\title{
Optical Fiber Sensors for Smart Composite Materials and Structures
}

\author{
Manjusha Ramakrishnan \\ Technological University Dublin, manjusha.ramakrishnan@mydit.ie \\ Yuliya Semenova \\ Technological University Dublin, yuliya.semenova@tudublin.ie \\ Gerald Farrell \\ Technological University Dublin, gerald.farrell@tudublin.ie
}

See next page for additional authors

Follow this and additional works at: https://arrow.tudublin.ie/engschelebk

Part of the Electrical and Electronics Commons

\section{Recommended Citation York, CRC Press. doi:10.13140/RG.2.1.2557.7687 \\ This Book Chapter is brought to you for free and open access by the School of Electrical and Electronic Engineering at ARROW@TU Dublin. It has been accepted for inclusion in Books/Book chapters by an authorized administrator of ARROW@TU Dublin. For more information, please contact arrow.admin@tudublin.ie, aisling.coyne@tudublin.ie, gerard.connolly@tudublin.ie.}

Ramakrishnan, M., Semenova, Y. \& Farrell, G. (2015). Optical Fiber Sensors for Smart Composite Materials and Structures. In Ginu Rajan (ed.), Optical Fiber Sensors: Advanced Techniques and Applications, New

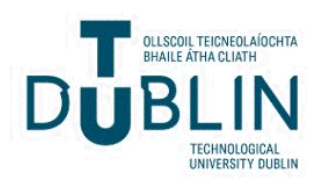


Authors

Manjusha Ramakrishnan, Yuliya Semenova, Gerald Farrell, and Ginu Rajan

This book chapter is available at ARROW@TU Dublin: https://arrow.tudublin.ie/engschelebk/16 


\title{
17 Optical Fiber Sensors for Smart Composite Materials and Structures
}

\author{
Manjusha Ramakrishnan, Yuliya Semenova, \\ Gerald Farrell, and Ginu Rajan
}

\section{CONTENTS}

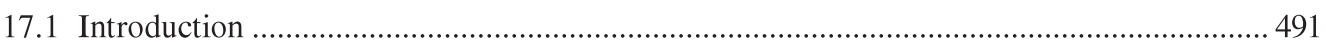

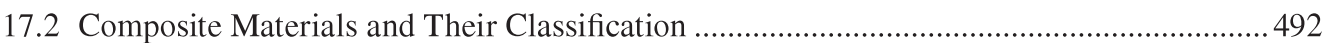

17.3 Demand for the SHM in Composite Material Structures ................................................. 494

17.4 Composite Materials with Embedded Fiber Sensors: Fabrication Methods ........................ 496

17.5 Composite Degradation Associated with Embedding of Fiber Sensors ............................. 496

17.6 Types of Optical Fiber Sensors for Composite Materials ............................................. 497

17.6.1 Fiber Bragg Grating Sensor for Composite Materials ....................................... 498

17.6.1.1 Strain and Temperature Measurements Using Embedded FBGs ..............500

17.6.1.2 Vibration Measurements Using Embedded FBGs .................................... 501

17.6.1.3 Effect of Thermal Expansion of the Composite Material on FBG

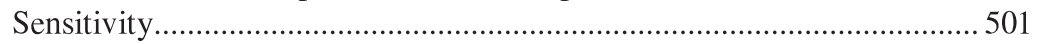

17.6.1.4 Acoustic Emission Measurements Using Phase-Shifted FBGs................502

17.6.2 FBG Written in Microstructured Fibers as a Sensor for Composite Materials ........ 502

17.6.3 Polymer FBG Sensor for Composite Materials .....................................................503

17.6.4 Interferometric Fiber Sensors for Composite Materials ..........................................506

17.6.5 Optical Fiber Polarimetric Sensors for Composite Materials ..................................508

17.6.6 Distributed Optical Fiber Sensors ..................................................................... 510

17.6.7 Hybrid Sensing Approaches for Simultaneous Strain and Temperature

Measurements in Composite Materials ...................................................... 512

17.7 Lightweight Sandwich Composites with Embedded Fiber Sensors .................................. 514

17.8 Recent Trends, Issues, and Future Challenges of the OFS Technology ........................... 516

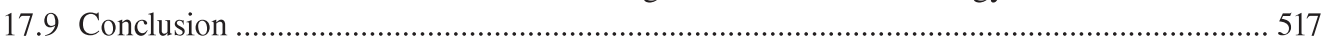

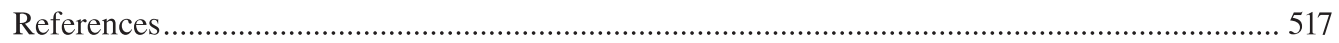

17.1 INTRODUCTION AQ1

Composite material structures [1] are widely used in the aerospace, marine, aviation, transport, sport/leisure, and civil engineering industries [1]. Such composite material structures are frequently subjected to external perturbations and varying environmental conditions, which may cause the structures to suffer from fatigue damage and/or failures, and thus require real-time structural health monitoring (SHM). Of necessity, the diagnosis process and condition monitoring of composite structures are usually carried out during their working life [2]. The goal of such diagnosis is to detect, identify, locate, and assess the defects that may affect the safety or performance of a structure. Sensors that are commonly employed for SHM are resistance strain gauges, optical fiber sensors 
(OFSs), piezoelectric sensors, eddy current sensors, and microelectromechanical systems (MEMS) sensors [3]. Traditional nondestructive evaluation (NDE) techniques such as ultrasonic inspection, acoustography, low frequency methods, radiographic inspection, shearography, acousto-ultrasonic, and thermography are effective in SHM of composite materials and structures, but it is difficult to use them in an operational structure due to the size and weight of the devices [4].

OFSs on the other hand are suitable candidates for SHM of composite materials during operation since they are capable of achieving the goals of diagnostics as well as condition monitoring and can also be embedded into such structures acting as a nervous system [5]. This powerful and potential rich technology is being currently implemented in a wide variety of applications. In the composite materials area, compared to traditional NDE techniques, OFSs offer unique capabilities: monitoring the manufacturing process of composite parts, performing nondestructive testing once fabrication is complete, and enabling health monitoring and structural control. Investigations of OFS embedded in composite structures indicate that OFS technology is capable of monitoring stress/ strain, temperature, composite cure process, vibration, humidity, delamination, and cracks and thus has great potential for condition monitoring of a variety of composite materials applications [6,7]. Because of their minimal weight, small size, high bandwidth, and immunity to electromagnetic interference, OFSs have significant performance advantages over traditional sensors. Furthermore, optical fibers are steadily becoming more cost-effective due to advances in the telecommunication and optoelectronic industries. Also, to ensure the integrity of composite structures, an important requirement for the sensor is that it should be possible to embed it into the host composite without modifying the host's properties and functions. This can be achieved by OFSs, which are compatible with reinforcement fabrics and can be embedded within the structure without affecting its strength and thereby act as the nerves of the system and provide vital information on the internal mechanical health of the structure.

Recognizing the increase in the use of composite structures, this chapter describes an overview of recent advances in the area of OFS technology for condition monitoring applications in composite materials, arranged as a number of sections. An overview of different types of most commonly used composite materials and their properties is given in Section 17.2. Then the demand for the SHM in composite material structures is discussed in Section 17.3, following which the common fabrication methods of composite materials with embedded fiber sensors are detailed in Section 17.4 while the issues of composite degradation associated with embedding of fiber sensors are discussed in Section 17.5. An overview of different types of fiber sensors for strain/temperature measurements in composite materials is presented in Section 17.6 and an introduction to lightweight composite material is presented in Section 17.7. The recent trends, issues, and future challenges of the OFS technology are discussed in Section 17.8. The aim of this chapter is to provide a state of the art in the area of composite materials embedded with OFSs and its potential application in SHM.

\subsection{COMPOSITE MATERIALS AND THEIR CLASSIFICATION}

In this section, we discuss important classifications of typical composite materials and their properties. In general, fiber composite materials have two constituents: reinforcement and matrix [8], which when combined together can produce a material with properties superior to those of the constituent materials. It is known that the mechanical properties of the composite materials differ depending on the matrix and the holding reinforcing materials used to fabricate the composite material [8].

The functions of the matrix in a composite material are to transfer the load to the reinforcement fibers, provide temperature resistance and chemical resistance, and maintain the reinforcement fibers in a fixed orientation [9]. Matrix materials are generally divided into two categories: thermosets and thermoplastics [10]. Thermoset matrices are the most common, being low cost and solvent resistant. The curing process in the thermosets is an irreversible chemical process. The most common type of thermoset is epoxy resin, which possesses superior performance and is relatively low cost. 
Thermoplastic-based matrices can be softened by heating to an elevated temperature. Then to form a thermoplastic composite material, the softened matrix is mixed with the reinforcement fiber, a process that is reversible. The most common thermoplastic is polypropylene, which has a limited temperature range of up to $150^{\circ} \mathrm{C}$. The typically used thermoset and thermoplastic matrices are classified according to the extent of their use in Figure 17.1a and b, respectively.

The second constituent of a composite material is the reinforcement fiber [8]. The composite material's tensile properties, stiffness, and impact resistance are influenced by the type of the fiber reinforcement [9]. Thus, for a manufacturer, it is important to select an appropriate fiber reinforcement taking into account the end user's application of the fabricated composite structure. The reinforcement fiber materials are mainly categorized into two types: glass fiber and advanced fiber [10]. There are different kinds of glass fibers available that are made of different kinds of glass, ranging from normal silica glass to high purity quartz glass, and each of these glass fibers offers its own set of properties. There are also advanced fiber materials that offer high strength and high stiffness but with low weight [10]. Boron, silicon, carbide, carbon, Aramid (Kevlar ${ }^{\circledR}$ or Twaron ${ }^{\circledR}$ ), polyamide, nylon, self-reinforced polymer fibers (e.g., Curv ${ }^{\circledR}$, Pure ${ }^{\circledR}$, Tegris ${ }^{\circledR}$ ), basalt fiber, and natural fibers (e.g., flax, jute, coir, hemp, cotton) are examples of such advanced fibers. These reinforcement fibers can be arranged in a woven, knitted, braided, or nonwoven style. In the case of a multilayered composite material fabricated using woven-style reinforcement fiber fabrics, it is found that the mechanical properties are also determined by the fiber reinforcement fabrics' woven style, density, and ply configuration [7,8]. Typically available categories of composite material are shown in Figure 17.2.

As regards applications, fiber-reinforced composite materials are commonly used for fabrication of various structural parts such as aircraft tails, wings, fuselages, propellers, helicopter rotor blades, wind turbine blades, and parts of racing cars, boats, etc. [7]. One of the prominent challenges for a composite structural part designer is a fail-safe design solution, which requires optimization of multiple parameters. Typically, given the performance specifications for the composite part [8], the areas that need optimization are the following: the selection of most appropriate

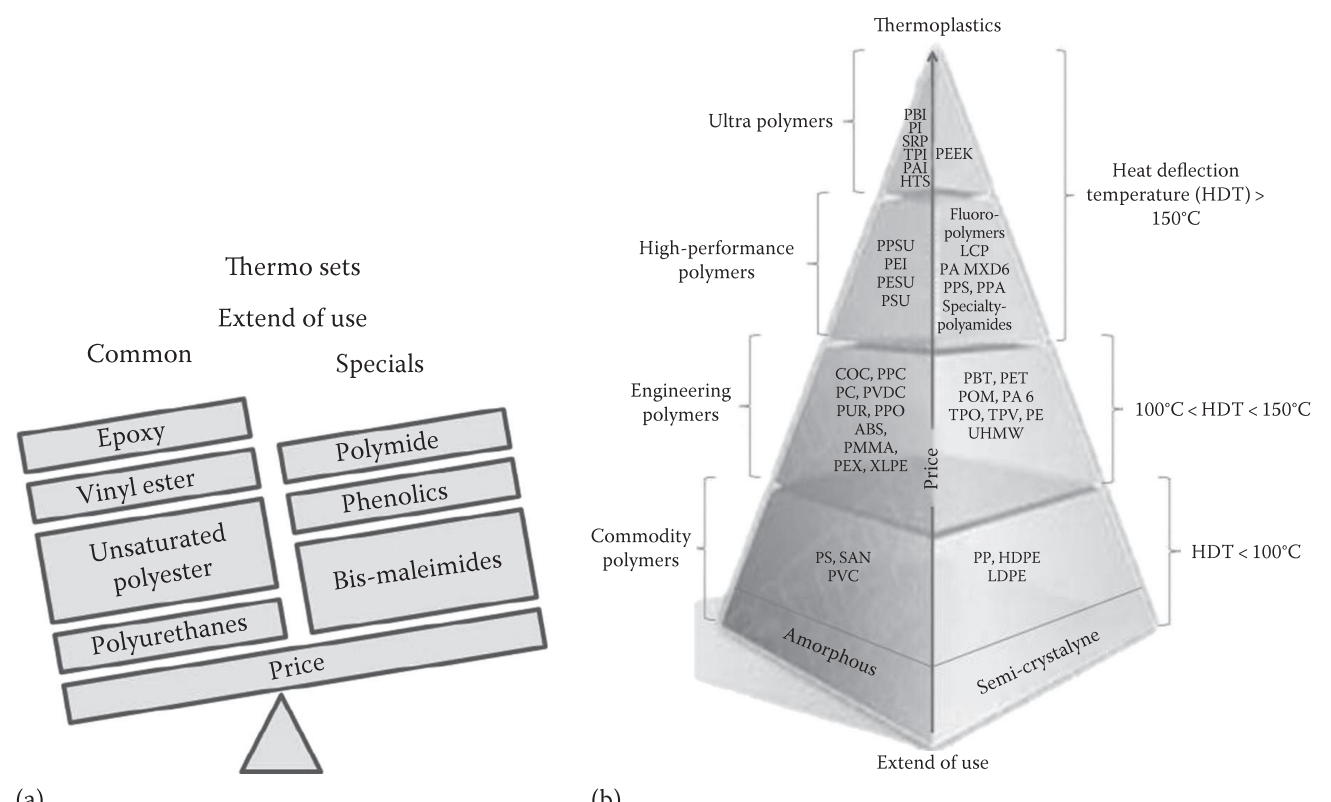

(a)

(b)

FIGURE 17.1 Classification of typical thermosets (a) and thermoplastic matrices (b) and the extent of their AQ3 use and price. (Courtesy of the special chem 12/08.) 


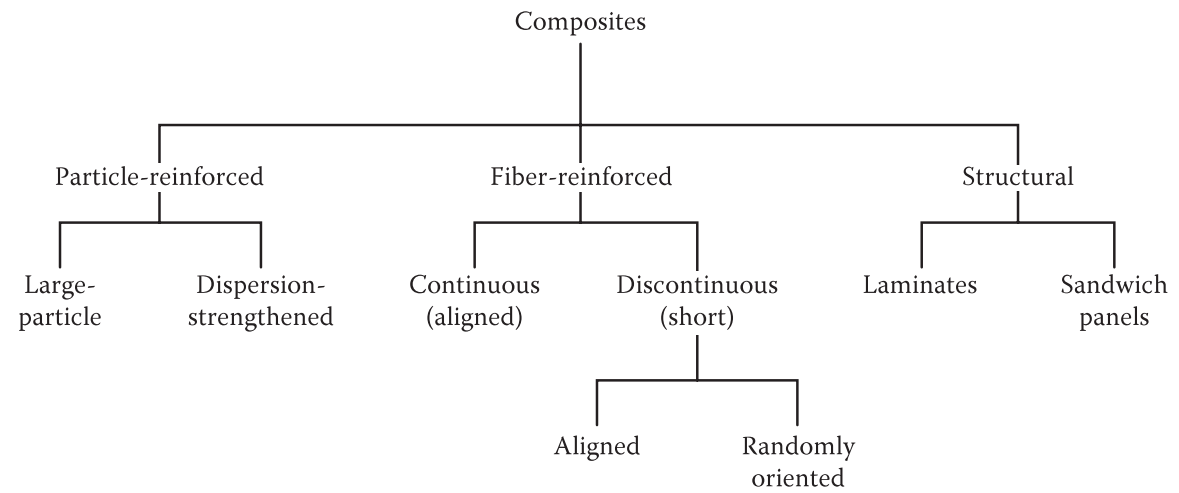

FIGURE 17.2 Typically available categories of composite materials. (From www.engineeringcivil.com.)

reinforcement and matrix constituents to satisfy the requirements for strength and stiffness of the particular composite part with a minimum weight; the selection of the composite part's geometry; a careful analysis of stress distribution within the part; the minimization of moisture ingress; optimization of toughness; and an analysis of failure modes. Prior to opting for a particular design for mass production, the manufacturer also needs to consider the repeatability of the composite part's performance specification, ease of production, cost efficiency, and quality assurance mechanisms $[9,10]$. Finally, even though the designed structural part is optimized for fail-safe performance, there is a possibility of damage during operation in extreme environmental conditions and mechanical failure of the structure due to external perturbations [3]. All of this necessitates the requirement for nondestructive SHM techniques throughout the lifetime of the composite structural part.

\subsection{DEMAND FOR THE SHM IN COMPOSITE MATERIAL STRUCTURES}

The demand for SHM in composites is in the first instance driven by the increased use of composite materials. One of the main reasons that drive industry to use composite materials is the benefit of lower weight, resulting in improved energy efficiency and less $\mathrm{CO}_{2}$ emissions. This increasing use of composite materials brings with it a need to establish inspection and/or monitoring regimes to ensure structural integrity and safe operation throughout service life. This can make the cost of ownership high, with downtime being an important issue [11]. Therefore, any SHM methodology that could increase the inspection intervals for a structure and indicate damage before costly failures occur would be very advantageous, financially and in many cases in terms of safety.

The global use of carbon fiber-reinforced plastics (CRP) and glass fiber-reinforced plastic (GRP) composite materials based on structural parts has significantly increased in application areas such as aircraft, sport vehicles, wind turbines, and infrastructure constructions, because of the inherent advantages of composite materials. For example, in aircraft, the advantages are well known: lighter weight for the aircraft, reduced requirements for maintenance, and increased passenger comfort. The use of composite materials in aircraft is growing as shown in Figure 17.3a. Composite-based aircrafts consume less fuel and result in a high cost benefit that yields energy savings up to $18 \%$. Some existing commercial aircraft models such as the Boeing 787 and Airbus A350 are comprised of circa $50 \%$ composite materials by weight. A market research conducted in 2013, in terms of the global CRP market by Bernhard Jahn and the European GRP market by Dr. Elmar Witten, indicates that there will be a growing demand for such aircraft in the coming years [12]. Figure 17.3b and c

AQ5 show the overall use of CFP composites by different industries and GRP production in different European industries, respectively. 


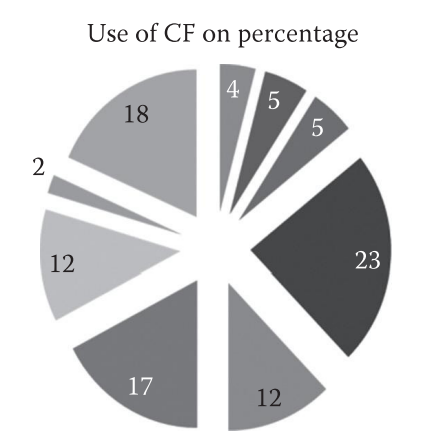

\begin{tabular}{|l|}
\hline Civil engineering \\
$\square$ Automotive \\
Molding and compoure vessel \\
\hline
\end{tabular}

(a)

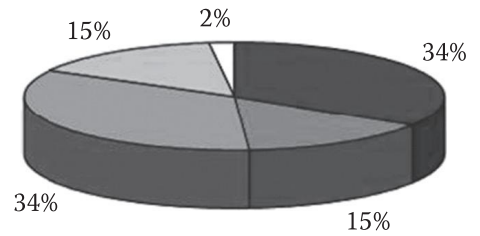

\begin{tabular}{|ll|}
\hline$\square$ Transport & $\square$ Electro/electronic \\
$\square$ Construction & $\square$ Sport and leisure \\
$\square$ Others & \\
\hline
\end{tabular}

(b)

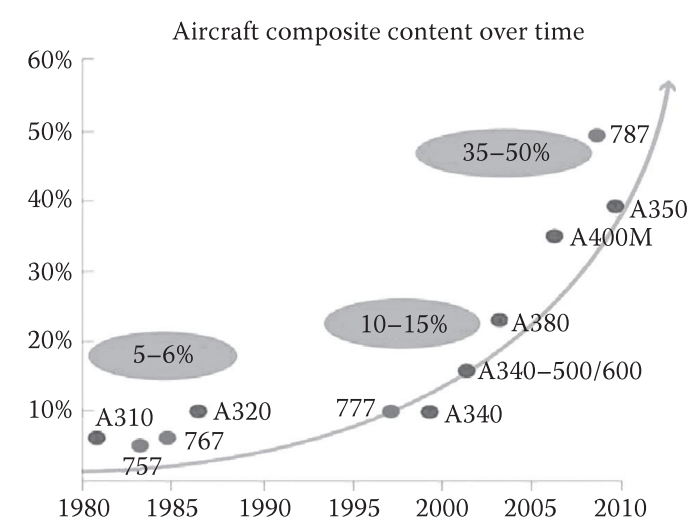

(c)

FIGURE 17.3 (a) Use of CF composites by industry (Courtesy of the global CRP market by CCeV), (b) GRP production in Europe for different application industries (Courtesy of the European GRP Market by AVK), and (c) development of composite aerospace applications in the last 40 years. (Courtesy of the Hexcel Corp. Aerostrategy.)

The second driving force behind the need for SHM in composite material is the necessity to monitor composite parts during operation, for safety and early detection of failure. During a typical 20-year service life, composite structures such as wind turbine blades, helicopter blades, and aircraft parts are subjected to static and dynamic lifts, and drag and inertial loads over a wide range of temperatures and often severe environmental conditions. As the growth of production of composite parts has increased, the composite industry has increasingly focused on damage/failurefree composite structures and nondestructive techniques for SHM over their lifetime. Compared to conventional nondestructive sensing techniques, OFSs have achieved wide acceptance due to their attractive properties such as small size, immunity to electromagnetic interference, and low cost [1]. OFSs embedded in various structures are very useful for strain/temperature monitoring [2] applications in extreme environmental conditions. For example, issues such as bend loading and icing can be monitored and avoided by implementing smart composite structures with embedded OFSs. Such smart composite materials with embedded OFSs can enhance the safety of advanced machines, structures, and devices. 


\subsection{COMPOSITE MATERIALS WITH EMBEDDED FIBER SENSORS: FABRICATION METHODS}

In this section, we discuss the commonly employed sample fabrication methods for composite materials with embedded OFSs. The fabrication processes commonly adopted by laboratory-based and small-scale industrial manufacturers are the expertise-intensive hand layup and pre-preg layup [13] methods. The hand layup method is a process for arranging fiber-reinforced layers in a laminate and shaping the laminate to fabricate the desired part. For this, the reinforcement fibers or fabrics are stacked one over another by applying the matrix in between them. After stacking, curing (or polymerization) of the resin matrix of the multiple laminate layered composite, the sample can be shaped as per the manufacturer's specifications. Pre-pregs are single laminates of pre-impregnated composite fibers with a matrix material such as epoxy resin. In the pre-preg layup method, multiples of composite pre-preg laminates are stacked one over another and the curing can be done unaided or by applying heat and/or pressure. The molding process can be either vacuum-bag molding or an autoclave molding. Typical autoclave curing conditions involve a temperature range from $120^{\circ} \mathrm{C}$ to $200^{\circ} \mathrm{C}$ and with an applied pressure up to 100 psi.

For embedding optical fibers in samples prepared by both the hand layup and the pre-preg layup methods, the process is similar: before the curing process, optical fibers are placed on the corresponding composite layer and some prestrain is applied to make sure the optical fibers remain free of bends [14]. Positioning of the optical sensor is highly application specific and depends on the location of the areas where parameters need to be monitored. For example, for strain and temperature measurements of composite material structures, it is reported that OFSs were embedded within the farthest layer from the middle layer to achieve the highest measurement sensitivity [15]. The different steps for embedding OFSs by the hand layup and pre-preg methods are shown in Figure 17.4a and b, respectively. For larger composite parts, optical fibers can be embedded manually with expert assistance as shown in Figure 17.4c, but for large-scale production of smart composite structure materials embedded with OFSs, automated robotic systems are now common and an example of such is a robotic system by SABCA Limburg and Sonaca/Ingersoll shown in Figure 17.4d. One of the most important concerns while embedding OFS inside composite structures is their influence on the structural integrity of the composite part. In the next section, we have detailed some methods to reduce the risk of composite material degradation and to maintain the structural integrity of composite material structures.

\subsection{COMPOSITE DEGRADATION ASSOCIATED WITH EMBEDDING OF FIBER SENSORS}

One of the major concerns of OFS for composite materials is the potential for degradation of the composite material mechanical properties and possible increased failure rate due to the presence of an embedded optical fiber [16]. Various studies have been carried out to analyze the influence of the embedded OFS on composite material tensile/compressive strength, stiffness, interlaminar fracture toughness, and fatigue resistance. Lee et al. reported that having embedded optical fibers passing through or parallel to ply drops in a laminate does not have any significant effects on the static strength of the laminate [17,18]. This is valid even for optical fibers placed in the most critical locations. In principle, any potential degradation of strength and the modulus of a composite material will be a function of the orientation of the optical fiber relative to the nearest plies, the overall thickness of the laminate, the optical fiber diameter, and the type of protective coating on the optical fiber [17]. Degradation becomes increasingly severe with an increasing angle between the optical fiber and ply directions. Another issue is the larger diameter of the optical fiber (with its buffer coating) compared to the ply fibers of the composite. In general, commercially available optical fibers have diameters from 125 to $230 \mu \mathrm{m}$, which is about 10-15 times larger than the average E glass fiber or carbon fiber. The optical fiber orientation perpendicular to the fiber reinforcement 


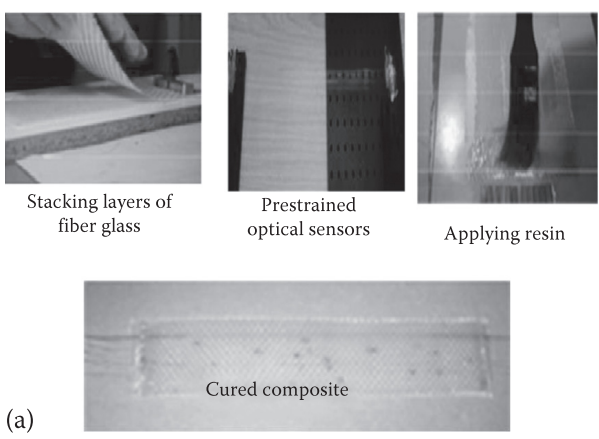

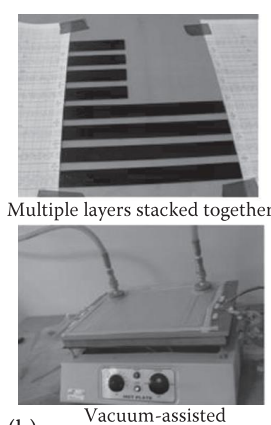

(b) high-temperature curing

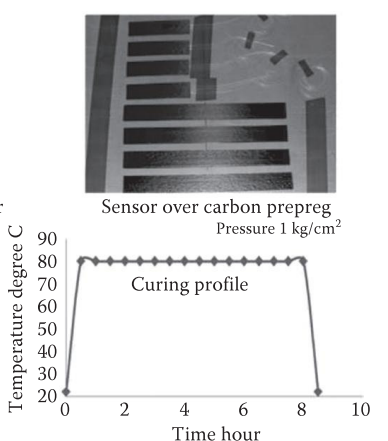

Time hour

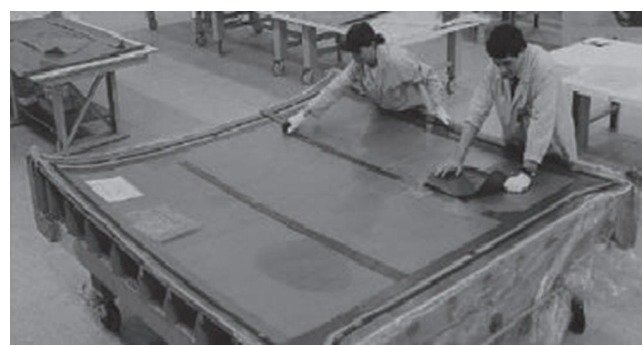

(c)

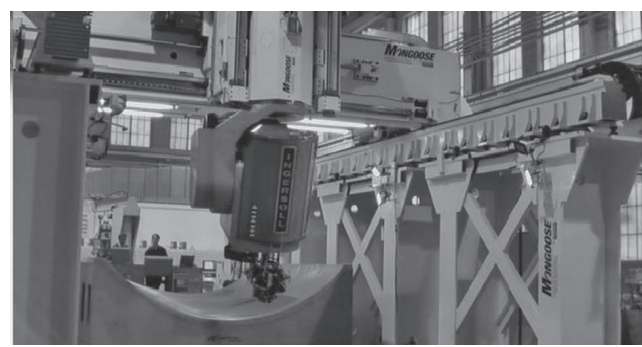

(d)

FIGURE 17.4 Embedding fiber sensors inside composite materials (a) hand layup, (b) pre-preg method, (c) expert-assisted manufacturing of composite part embedded with OFS, and (d) automated robotic-assisted manufacturing of composite structures embedded with OFS. (Photo (d): SABCA Limburg and Sonaca/ Ingersoll.)

can result in a characteristic eye pattern or pocket within the resin, which acts as a defect center in the composite part that could lead to premature failure in the form of delamination [18]. However, testing conducted at a number of labs worldwide concluded that such delaminations were insignificant if the OFS density was low as shown in Figure 17.5. Wojciech et al. reported that if the optical fiber is laid along the direction of reinforcement fiber, there is a uniform consolidation around the optical fiber with minimum defects and thus the laminate's mechanical parameters are least affected $[6,17,18]$. Also the optical fibers laid along the direction of fiber reinforcement have limited influence on the mechanical behavior of the composite structure because of the optical fiber's own inherent load carrying ability [17].

An analysis [19] on the flexural strength of a composite material, embedded with OFSs, that bears tensile loading showed that the flexural strength did not suffer any noticeable degradation when the OFSs were embedded in the tensile region either in the longitudinal direction or in the transverse direction. For compressive loading, the situation is similar if the sensor is embedded in the longitudinal direction but it is found that if the OFS is embedded in the transverse direction with
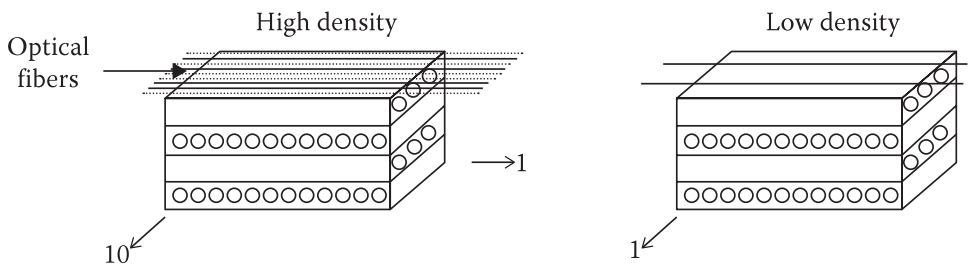

FIGURE 17.5 Optical fibers in composite materials embedded with high and low density. 
respect to the compressive region, the flexural strength is degraded significantly [21]. In order to realize SHM for composite material components in many applications, it is essential to measure the strain and temperature of composite materials, and in the next section we discuss the different types of OFS strain and temperature sensors that can be embedded in composite materials.

\subsection{TYPES OF OPTICAL FIBER SENSORS FOR COMPOSITE MATERIALS}

There are a wide variety of condition monitoring applications in composite materials, including cure process monitoring, vibration measurements, and detection of delamination and cracking. [20,21]. For all these applications, the key requirement is to measure either strain or temperature or both parameters. In this section, we discuss the different types of OFSs that can be used with composite materials to measure strain/temperature when embedded inside a composite material. The different types of OFSs reported for strain/temperature measurements for composite materials are fiber Bragg grating (FBG) sensors [22], interferometric OFSs [23], polarimetric sensors [24], distributed sensors (using techniques such as Rayleigh scattering, Raman scattering, and Brillouin scattering) [25,26], and hybrid sensors [20,21,27,28].

\subsubsection{Fiber Bragg Grating Sensor for Composite Materials}

FBGs are the most commonly employed OFSs in SHM applications for composite materials [22,29]. An FBG sensor comprises of a grating region with a periodic change in refractive index in the core region of an optical fiber. Such a periodically modulated refractive index structure enables the light to be coupled from the forward propagating core mode into a backward propagating core mode generating a reflection response, as shown in the schematic illustrating the principle of an FBG provided in Figure 17.6 [30].The light reflected due to periodic variations of the refractive index of the Bragg grating with a central wavelength is given by

$$
\lambda_{B}=2 n_{0} \Lambda
$$

where

$n_{0}$ is the effective refractive index of the fiber core

$\Lambda$ is the grating pitch

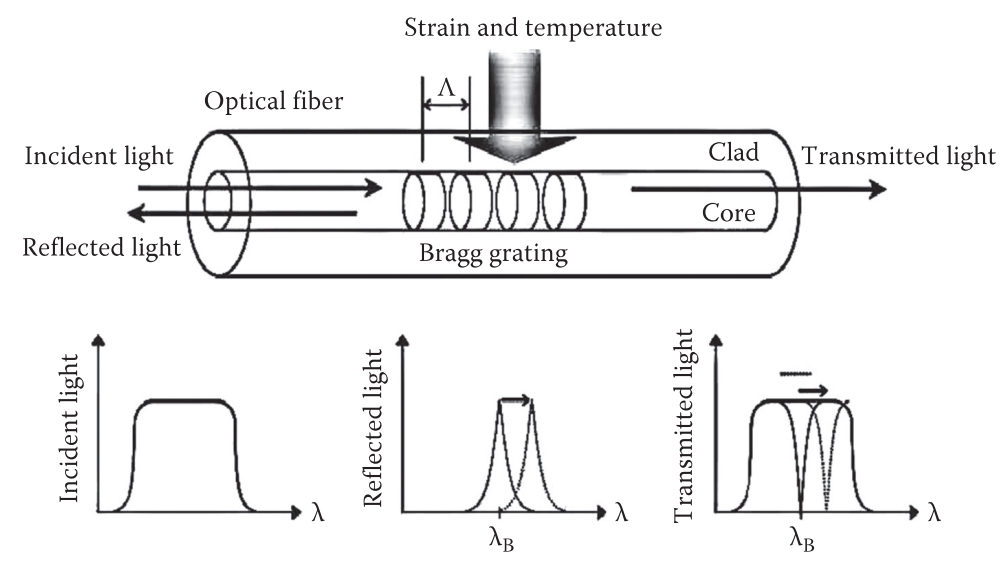

FIGURE 17.6 FBG. 
The strain sensitivity of the Bragg wavelength arises from the change in the pitch of the fiber grating due to the strain and changes in the refractive index arising from the strain-optic effect. When a strain is applied to the grating, the Bragg reflected wavelength changes. The wavelength shift $\Delta \lambda_{\varepsilon}$ for a value of elongation $\Delta L$ is given by

$$
\Delta \lambda_{\varepsilon}=\lambda_{B} \cdot\left(\frac{1}{\Lambda} \frac{\partial \Lambda}{\partial L}+\frac{1}{n_{0}} \frac{\partial n_{0}}{\partial L}\right) \cdot \Delta L
$$

In practice, the applied strain value can be estimated from the measurement of reflected wavelength as it changes due to the applied strain. The typical strain sensitivity of an FBG at $1550 \mathrm{~nm}$ is $\sim 1.2 \mathrm{pm} / \mu \varepsilon$.

Bragg wavelength shift can also occur due to changes in temperature. For a temperature variation of $\Delta T$, the corresponding wavelength shift $\Delta \lambda_{T}$ is given by

$$
\Delta \lambda_{T}=\lambda_{B} \cdot\left(\frac{1}{\Lambda} \frac{\partial \Lambda}{\partial T}+\frac{1}{n_{0}} \frac{\partial n_{0}}{\partial T}\right) \cdot \Delta T
$$

The temperature sensitivity of the Bragg wavelength arises from the change in the grating pitch associated with the thermal expansion of the fiber and the change in the refractive index arising from the thermo-optic effect. Thus, Equation 17.3 can also be written as

$$
\Delta \lambda_{T}=\left(\alpha_{0}+\beta_{0}\right) \cdot \lambda_{B} \cdot \Delta T
$$

where

$\alpha_{0}$ is the coefficient of thermal expansion (CTE) of the fiber

$\beta_{0}$ is the fiber refractive index variation with temperature

The values of $\alpha_{0}$ and $\beta_{0}$ are constants for silica optical fiber and are $0.55 \times 10^{-6} /{ }^{\circ} \mathrm{C}$ and $6.6 \times 10^{-6} /{ }^{\circ} \mathrm{C}$, respectively. The typical temperature sensitivity of an $\mathrm{FBG}$ at $1550 \mathrm{~nm}$ is $\sim 11.6 \mathrm{pm} /{ }^{\circ} \mathrm{C}$.

\subsubsection{Strain and Temperature Measurements Using Embedded FBGs}

Commonly, FBGs are used to measure axial strain in the longitudinal direction as this provides higher sensitivity and temperature. For example, the measured wavelength shift of the embedded FBG sensors placed at different locations inside the composite for different deflection values during a three-point bending strain test on composite material is shown in Figure 17.7a. The measured wavelength shift of the embedded FBG sensors at different temperatures of composite materials is shown in Figure 17.7b. One can see that a red shift of the Bragg wavelength arises as a result of an increase in temperature. Other than standard Bragg gratings with uniform period, there are chirped gratings with a gradual period variation [31], and tilted fiber Bragg gratings (TFBG) [31] with gratings written at an angle to the fiber axis are also employed for strain temperature measurements in composite materials. The chirped FBG sensor has a gradual distribution of the grating period [32]. This variation in the grating period provides a one-to-one correspondence between the wavelength in the spectrum and the position of the gauge section within the composite material, which is the significant advantage of chirped FBG sensors over the conventional FBG sensors [32]. The main disadvantage of conventional FBG sensors is the cross-sensitivity between temperature and strain. In a different manner from conventional FBGs, the wave vector of a TFBG has a certain angle with respect to the fiber axis, making the resonance wavelengths of core mode and cladding mode to be sensitive to temperature, while their transmission power is temperature independent [33]. Thus, based on these unique characteristics of TFBG, it is possible to achieve 

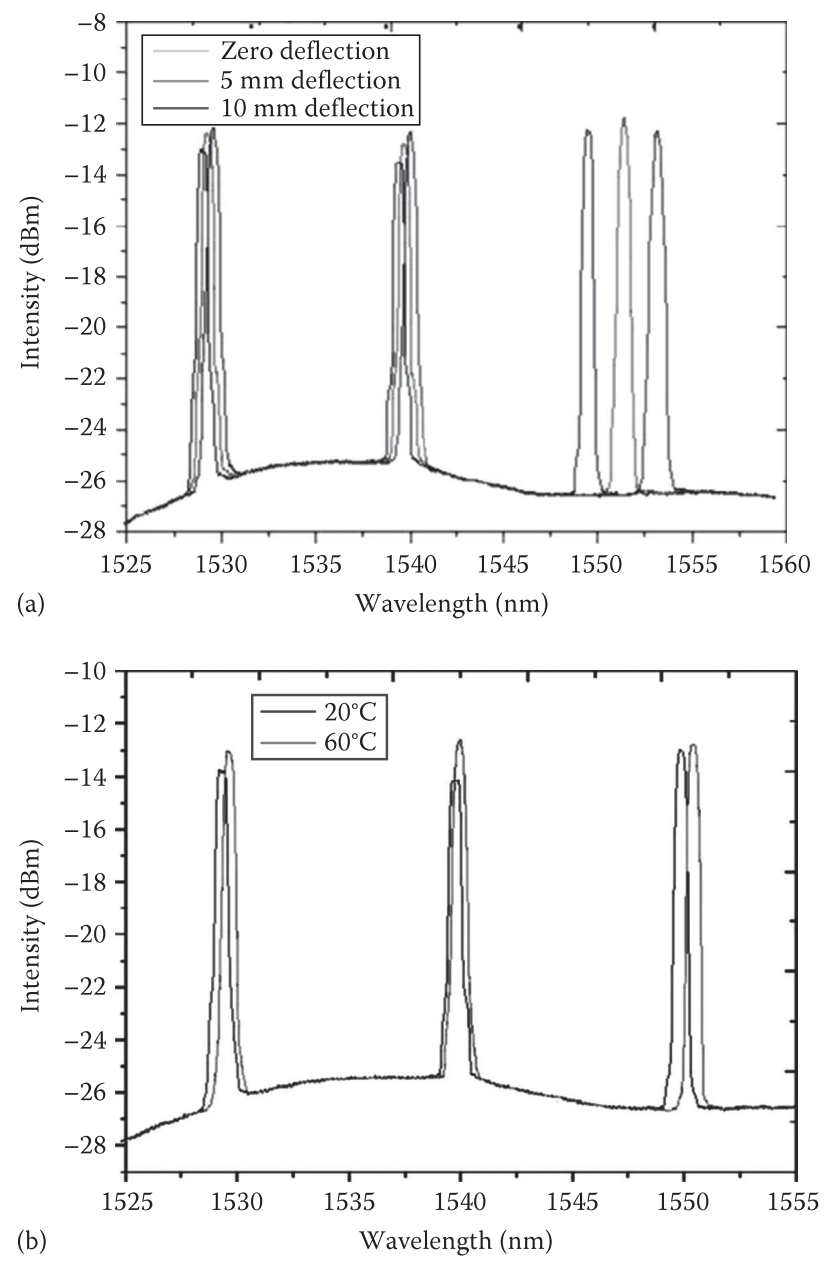

FIGURE 17.7 Wavelength shift for the FBG sensors embedded in composite materials at different locations (a) at different deflection values and (b) at different temperatures.

simultaneous discrimination of mechanical perturbations and temperature [31,33]. Different methods have been reported by other authors to compensate for the cross-sensitivity effects between temperature and strain; these methods include introduction of a reference FBG [34], dual-wavelength superimposed FBGs [35], combined FBG and a long-period grating (LPG) [36], different cladding diameter FBGs [37], combination of an FBG and a Fabry-Perot interferometer [38], and superstructure FBG method [39].

It is also reported that for coating-stripped FBGs, the transverse CTE for a composite material as well as the uniform lateral strain can cause Bragg peak distortion and broadening [40]. Such a nonuniform lateral strain distribution in the composite material can be measured by using an FBG written in a microstructured high-birefringence fiber [41].

\subsubsection{Vibration Measurements Using Embedded FBGs}

Composite material structures are frequently subjected to external excitations over a variety of vibration frequency ranges. Such dynamic interference may cause the structures to suffer from fatigue damage and/or catastrophic failures if the excitation frequency approaches natural 

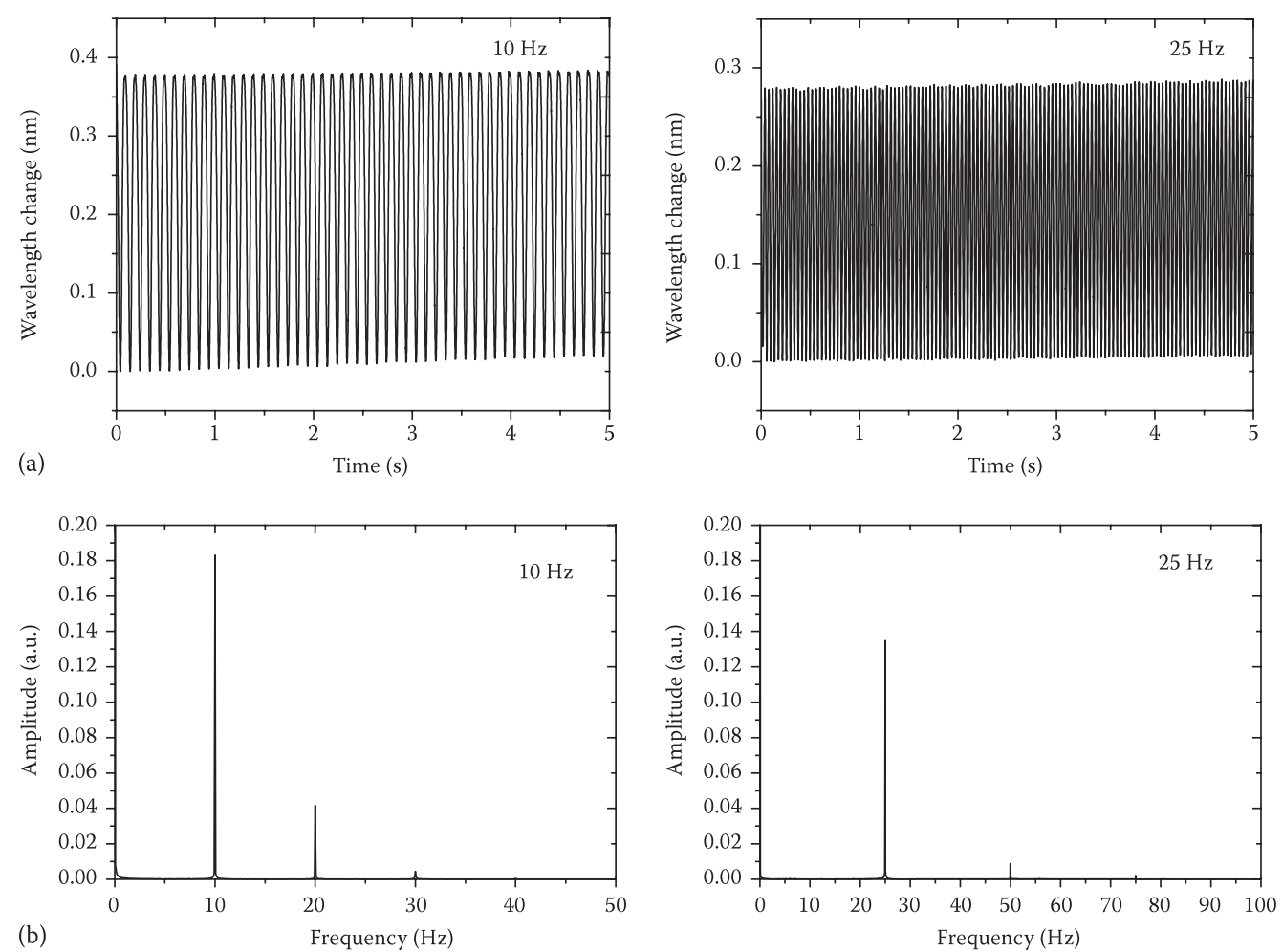

FIGURE 17.8 (a) Temporal and (b) FFT spectrum of the FBG sensor at different frequencies. (From Rajan, G. et al., IEEE Sensors J., 12(5), 1365, 2012.)

frequency of the structures. The microstructural mechanisms of damage accumulation include fiber breakage and matrix cracking, debonding, transverse ply cracking, and delamination. These mechanisms may occur independently or sometimes interactively, and the predominance of one or the other may be strongly affected by both material variables and testing conditions [42]. FBGs can be effectively used for vibration measurements of composite materials. Rajan et al. demonstrated that FBGs embedded in composite materials can be used to measure vibrations up to a frequency of $25 \mathrm{~Hz}$ [43]. The time-domain responses of the embedded FBG sensor at various frequencies are shown in Figure 17.8a and the corresponding frequency spectra are shown in Figure 17.8b.

\subsubsection{Effect of Thermal Expansion of the Composite Material on FBG Sensitivity}

For an FBG sensor embedded inside a composite material, three effects can take place due to change in temperature: (1) wavelength shift induced by the thermo-optic effect and thermal expansion of the polymer fiber; (2) wavelength shift induced by a longitudinal strain resulting from the thermal expansion of the composite sample; and (3) wavelength shift and spectral broadening corresponding to a transverse strain (or thermal stress) induced by thermal expansion of the composite sample. Due to the combined effect of (1) and (2), the temperature sensitivity for the embedded FBG sensor will be different from that for a free-space grating and if the transverse strain is effectively transferred to the grating, additionally a spectral broadening can be observed. Based on the type of reinforcement used, orientation of the reinforcement ply, and matrix materials, the magnitude of the wavelength shift due to thermal expansion may vary. 


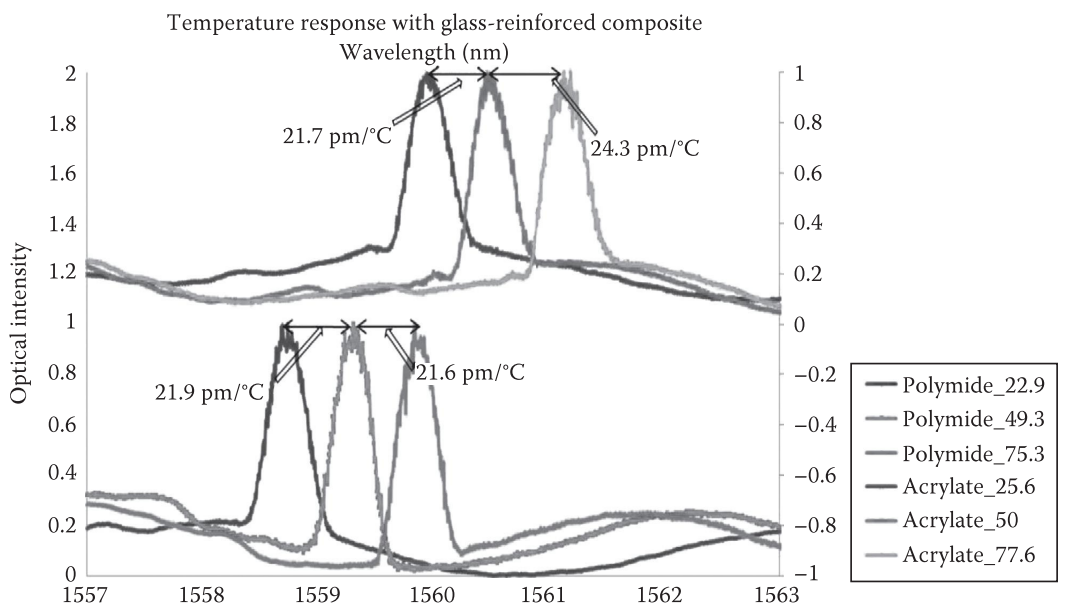

FIGURE 17.9 Comparison of wavelength shift of acrylate- and polyimide-coated FBGs embedded in glass fiber pre-preg composite material. (From Rajan, G. et al., Proc. IEEE Sens., 351, 2011.)

Considering only the temperature effect on the FBG and the thermal expansion-induced mechanical strain, the net wavelength shift for an embedded FBG with temperature can be written as [14]

$$
\Delta \lambda_{T}=\left(\alpha+\xi+k_{c}\right) \lambda_{B} \Delta T
$$

where

$\alpha$ is the CTE

AQ6 $\xi$ is the thermo-optic coefficient of the optical fiber

$\lambda_{B}$ is Bragg wavelength of the FBG

The coefficient $k_{c}$ corresponds to the effective mechanical strain on the FBG due to the thermal expansion of the composite material

Figure 17.9 shows the temperature sensitivity of the embedded silica FBGs with polyamide and acrylate coating, which shows significantly high temperature sensitivity compared to the free-space temperature sensitivity that arises from the strain induced on the grating due to thermal expansion.

\subsubsection{Acoustic Emission Measurements Using Phase-Shifted FBGs}

Among the FBG-based sensing techniques for acoustic emission (AE) measurement, one of the proven and most reliable techniques is based on phase-shifted FBG (PS-FBG) sensors and its corresponding interrogation system. PS-FBGs are widely used in optical fiber communications and optical fiber sensing applications as a wavelength multiplexer and also as a strain sensor. The extremely sharp resonance in the transmission spectrum of the PS-FBG enables it to develop a high sensitivity interrogation system capable of measuring very small strain changes, even at high acoustic frequencies. A typical transmission spectrum of a PS-FBG interrogated using a tunable laser from is shown in Figure 17.10. Using this method, it is proved that microscale strain can be measured and is suitable for AE sensing in composite structures [45].

\subsubsection{FBG Written in Microstructured Fibers as a Sensor for Composite Materials}

AQ7 Conventional FBG sensor's primary response is to an axial strain and thus transverse strain has very little influence on such an FBG sensor's response. For strain mapping in some SHM applications, 


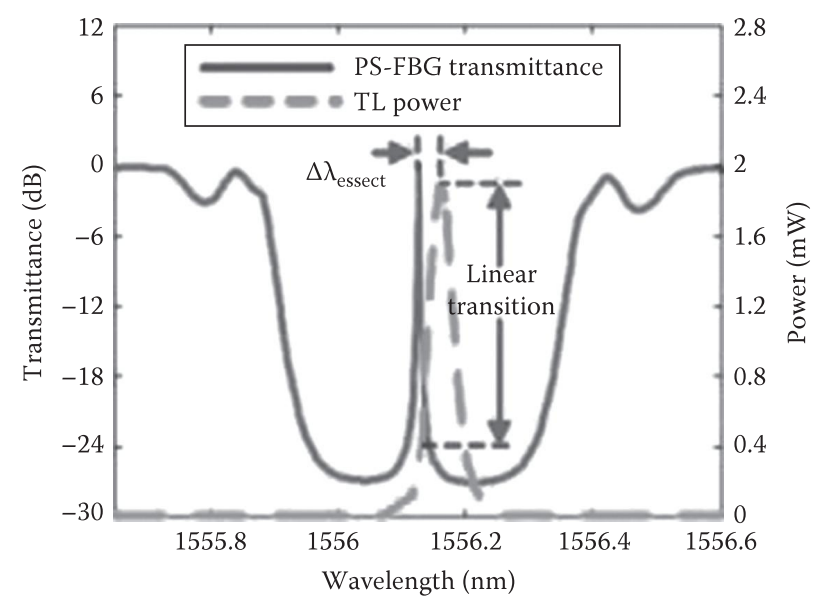

FIGURE 17.10 Spectral response of a PS-FBG and its interrogation technique based on a narrow band laser signal. (From Azmi, A.I. et al., Photon. Sens., 3(2), 184, 2013.)

for example, for the detection of damage, cracks, delamination, etc., a multiaxial strain measurement is required. This in turn results in the importance of development of a sensing scheme that provides measurements of axial strain together with lateral stain. Luyckx et al. reported that FBGs written in highly birefringent (HB) fibers and highly birefringent microstructured optical fibers (HB-MOFs) have real potential to measure transverse strain and axial strain simultaneously [41,46]. As represented in Figure 17.11a, an FBG written in an HB fiber displays two Bragg peaks, corresponding to both orthogonally polarized modes. The Bragg peak separation depends on the phase modal birefringence variation induced by transverse load and temperature. The properties of an MOF and its sensitivity to different measurands are determined by the type of the fiber used. But it is reported that FBGs written in HB fibers such as bow-tie type and Panda type have the disadvantage of greater temperature and strain cross-sensitivity.

The cross-sensitivity issue can be resolved by writing FBGs in low temperature-sensitive MOFs, such as highly birefringent polarization-maintaining photonic crystal fibers (HB-PM-PCF). For example, a comparison response of the embedded $\mathrm{FBG}$ sensors written in conventional birefringent optical fibers (bow tie) and an HB-PM-PCF when the composite material is exposed to controlled mechanical and thermal loads are presented in Figure 17.11b and c, respectively. For the FBG in a bow-tie-type fiber, the Bragg peak separation varies in accordance with transverse stain and temperature as shown in the figures. However, in the case of an FBG in an MOF, the Bragg peak separation varies with transverse stain only. A temperature-independent axial strain measurement in a composite material can be carried out using an FBG written in an HB-PM-PCF [46]. It is also reported that the two Bragg peaks in the case of FBG in PM-PCF are farther separated than for an FBG in a bow-tie fiber, and therefore FBGs written in MOF/PM-PCF allow for more accurate measurements of the peak wavelengths and thus are more suitable for composite material sensing applications [41,46].

\subsubsection{Polymer FBG Sensor for Composite Materials}

Polymer FBGs have attracted much interest among application engineers and scientists due to their unique advantages compared to the silica counterparts, such as the inherent fracture resistance, low Young's modulus, high flexibility, high temperature sensitivity, large strain measurement range, and low density [47-49]. In recent years, advancements in the fabrication of single-mode polymer optical fiber (POF) have also enhanced the research and development of Bragg grating sensors in 

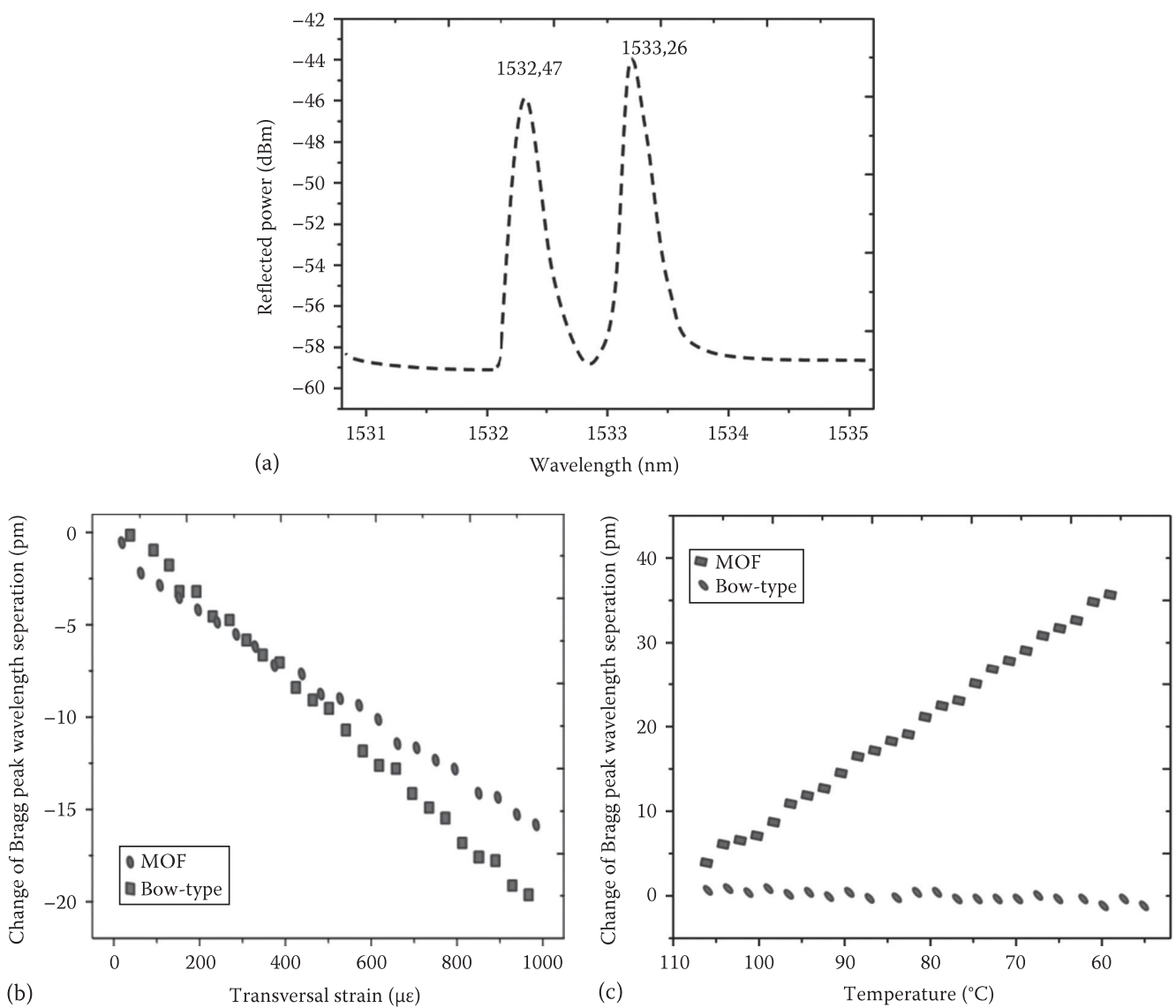

FIGURE 17.11 (a) Reflection spectra for an FBG written in an HB-PM-PCF with two peaks corresponding to slow axis and fast axis, (b) change in the peak separation with transverse strain for FBGs written in MOF and bow-tie-type fibers, and (c) change in peak separation with temperature. (From Luyckx, G. et al., IEEE Photon. Technol. Lett., 21, 1290, 2009.)

POF [50]. Sensors for different applications based on single-mode POF gratings have been reported [51,52]. For composite materials, the attractive characteristics of polymer FBGs include their high temperature sensitivity, large strain range, and the absence of buffer coating. These distinct features of the polymer FBGs might give an edge over the standard silica FBGs in measuring some of the parameters of the composite materials.

Rajan et al. conducted studies with polymer FBGs embedded in glass-reinforced composite materials [53]. For the polymer FBG embedded in composite materials, due to temperature change, spectral broadening is observed together with the shift in the peak reflected wavelength. The observed wavelength shift of the polymer FBG embedded in a glass-reinforced composite and its comparison with silica FBG and free-space FBG sensors are shown in Figure 17.12a. A blue shift in the wavelength is observed for the polymer FBG due to the negative thermo-optic coefficient, while a red shift is observed for the silica FBG. The observed temperature sensitivity of the embedded polymer $\mathrm{FBG}$ is $92.28 \pm 2 \mathrm{pm} /{ }^{\circ} \mathrm{C}$, which is close to the free-space temperature sensitivity of $90 \pm 6 \mathrm{pm} /{ }^{\circ} \mathrm{C}$.

The change in bandwidth of the reflection spectra of the polymer FBG at different temperatures and its comparison with silica FBG is shown in Figure 17.12b. It can be seen that the bandwidth of the polymer FBG increases as the temperature increases. The measured bandwidth change for the 

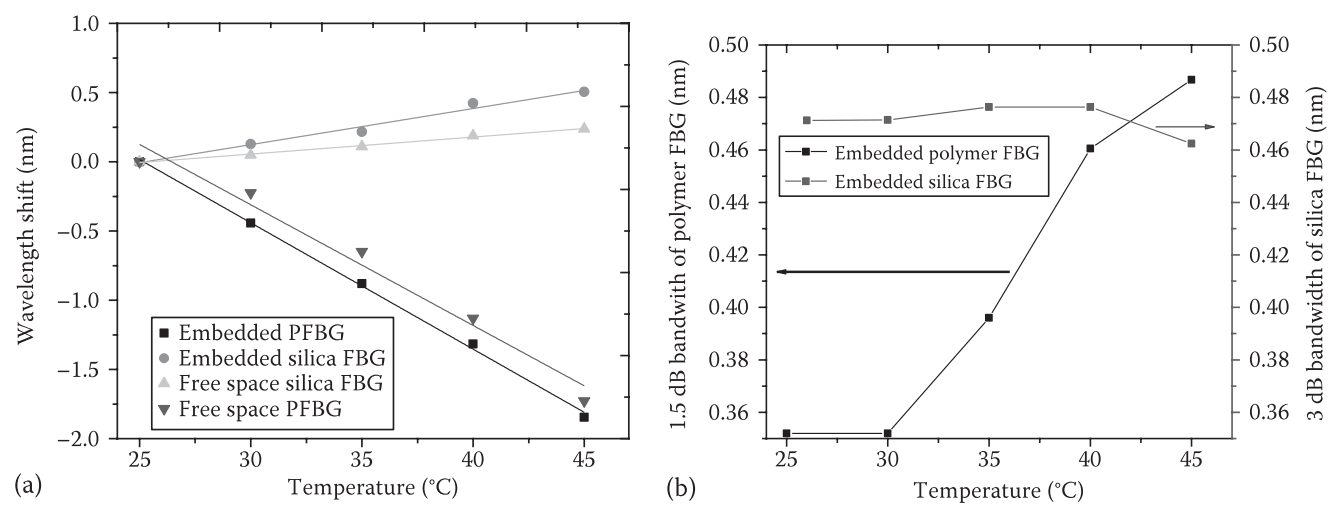

FIGURE 17.12 (a) Temperature-induced wavelength shift of the embedded polymer and silica FBGs and its comparison with free-space FBGs, (b) measured $1.5 \mathrm{~dB}$ bandwidth of polymer FBG and $3 \mathrm{~dB}$ bandwidth of silica FBG at different temperatures.

embedded polymer FBG within the temperature range of $30^{\circ} \mathrm{C}-45^{\circ} \mathrm{C}$ was $8.5 \mathrm{pm} /{ }^{\circ} \mathrm{C}$. Therefore, from the observed spectral broadening and distortion, it can be concluded that the thermal expansion induced stress is effectively transferred to the polymer fiber and can be measured using a polymer FBG. It is assumed that the reason for this is the absence of a buffer coating for the polymer fiber, which results to a direct transfer of the surrounding physical phenomena to the core and cladding of the polymer fiber. Stress induced by localized microbends in the composite material could also contribute to the chirping effect.

The strain sensitivity of the embedded polymer FBG and its comparison with silica FBG is shown in Figure 17.13. From the figure, it can be seen that the strain sensitivity of the embedded polymer and silica FBGs are very close to each other. In free space, the polymer FBG had a slightly higher strain sensitivity $(1.340 \pm 0.015 \mathrm{pm} / \mu \varepsilon)$ compared to that of silica FBG $(1.2 \pm 0.01 \mathrm{pm} / \mu \varepsilon)$. The similarity in the measured strain sensivity of the embedded silica and polymer FBGs underlines the fact that longitudinal strain in the composite is not effectively transferred to the polymer fiber. This can be attributed to the differnces in mechanical properties of the polymer fiber and the

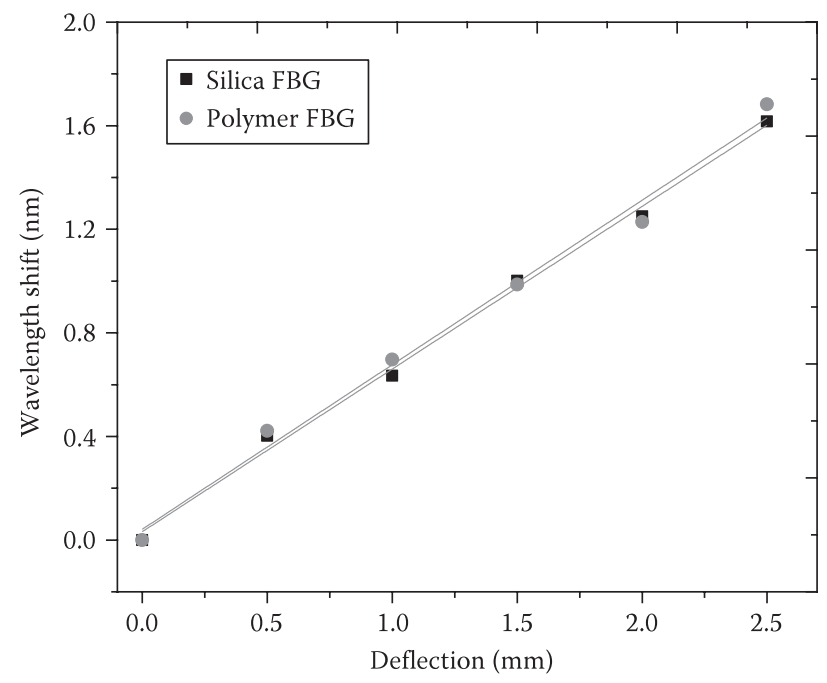

FIGURE 17.13 Wavelength shifts of the polymer and silica FBGs with deflection in the middle of the composite material. 
composite material, which resulted in the mechanical strain not being effectively transferred to the polymer FBGs as compared to the case of silica FBG. With further works in this area, polymer FBG has the potential in measuring temperature and thermal expansion of the composite material simultaneously.

\subsubsection{Interferometric Fiber Sensors for Composite Materials}

Interferometric fiber sensors [24] can also be employed for strain/temperature measurements in composite materials and this is discussed in detail in this section. There are different types of interferometric fiber sensors that differ in their operating principles and strain/temperature sensing characteristics. Among the interferometric fiber sensors most commonly employed for composite sensing applications are extrinsic Fabry-Perot interferometers (EFPI), microhole collapsed modal interferometers, and Sagnac fiber loop mirror (FLM) sensors.

The schematic experimental arrangement of the EPFI sensor is shown in Figure 17.14b, which comprises of a superluminescent light diode (SLD) source, a coupler, and a spectrometer. Any applied longitudinal strain to the FPI sensor alters the physical length of the cavity, which results in a phase difference between reflected or transmitted beams. By measuring the shift of the wavelength spectrum, the strain applied to the FPI can be measured. It is found that the shorter the optical path difference (OPD), the larger will be the free spectral range (FSR), resulting in a wider dynamic range for a sensor [24,54]. Therefore, the dynamic range of the sensor can be tuned by varying the cavity length, which in turn changes the OPD of the FPI sensor [54].

EFPI sensors based on PCFs are proved to be good candidates for measurements of axial strain in composite materials since their sensitivity to transverse strain and temperature is insignificant [55-57]. Figure 17.15a illustrates experimentally measured strain using embedded EFPI sensor during the three-point bending test of a composite material sample [57].

Recent advancements in the area of PCF sensors [58] have opened new possibilities for the development of temperature-insensitive microhole collapsed PCF modal interferometric sensors. Microhole collapsed PCF modal interferometers can be used for localized strain measurements in a composite material. It can be fabricated by fusion slicing of a PCF section between two standard single-mode fibers. During the splicing process, at both ends of the PCF section, the holes of the PCF are collapsed in a microscopic region. The first collapsed region allows for the excitation of multiple modes in the PCF and at the second collapsed region at the other end the modes recombine and thus the PCF section forms an interferometer [58]. Temperature-independent strain measurement in composites using such a microhole collapsed PCF interferometer fabricated from a microstructured PM-PCF fiber (LMA-10) was reported by Rajan et al. An example of the output of a microhole collapsed modal interferometer embedded in composite beam undergoing three-point

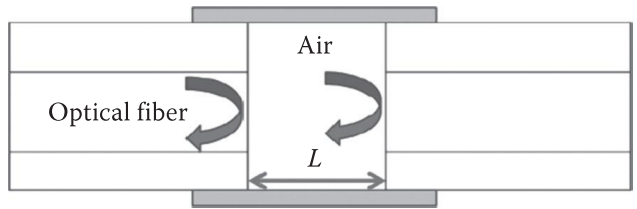

(a)

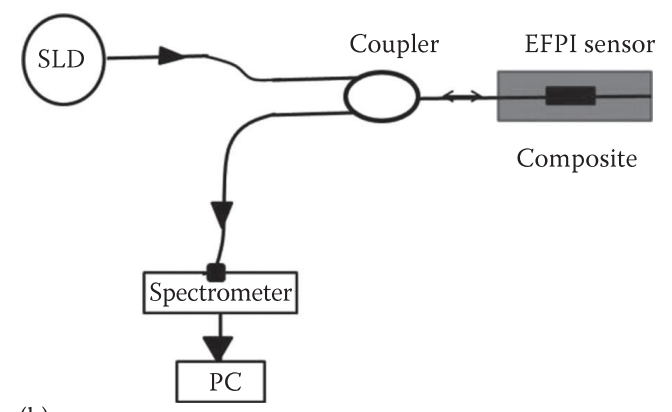

(b)

FIGURE 17.14 (a) One of the typical EFPI sensor design and (b) schematic experimental arrangement for the EPFI sensor. 

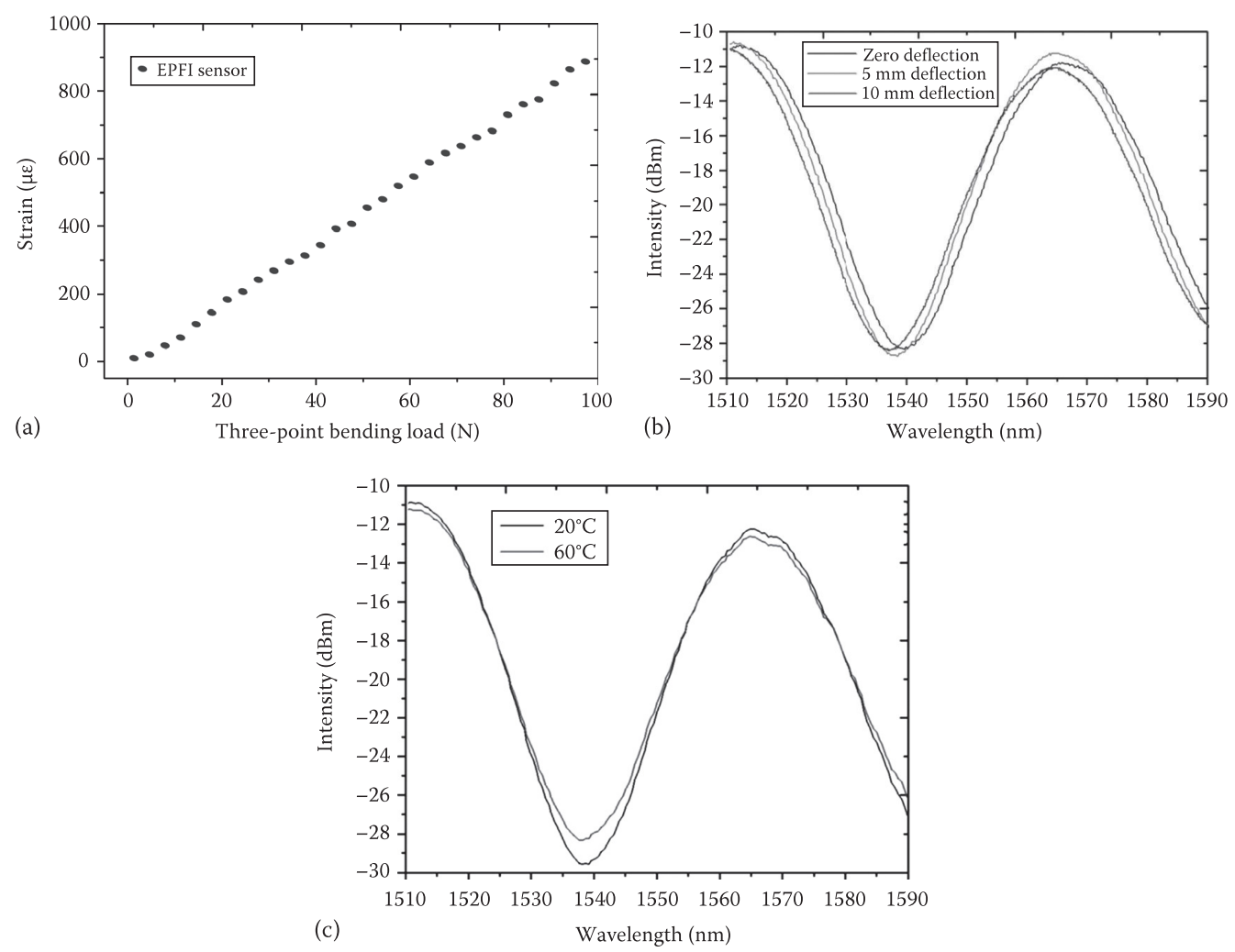

FIGURE 17.15 (a) Experimentally measured strain using embedded EFPI sensor during a three-point bending test in a composite material (From Leng, J.S. et al., Sensor. Actuat. A-Phys., 103, 330, 2003), (b) responses of the of PCF-based sensors embedded in the composite material sample during deflections based on a threepoint bending test, and (c) at different temperatures of the composite sample.

bending deflection is shown in Figures 17.14b and 17.15c illustrates that such a sensor embedded in composite material has very low temperature dependence [58].

Fiber-optic Sagnac interferometers (SIs) are another promising candidate for composite material sensing applications. In a fiber-optic SI, input light is split into two parts propagating in the opposite directions by a $3 \mathrm{~dB}$ fiber coupler, and these two counterpropagating beams are combined again at the same coupler as shown in Figure 17.16. The fabrication of such an interferometer can be

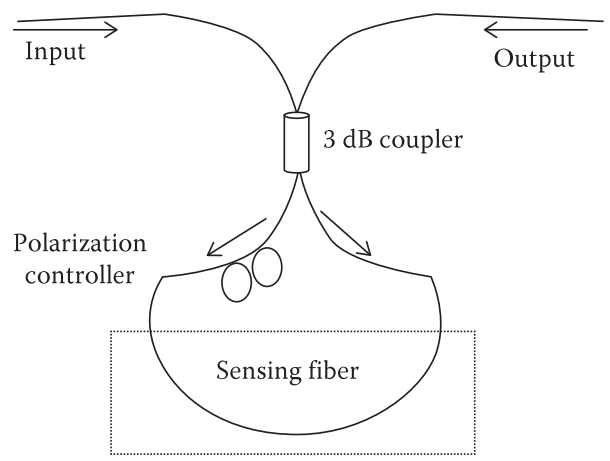

FIGURE 17.16 Schematic of the sensor based on an SI. 
simply achieved by connecting the ends of a conventional $3 \mathrm{~dB}$ coupler. HB fibers or polarizationmaintaining fibers (PMFs) are typically utilized as sensing fibers since HB fibers maximize the polarization dependence of the signal within the SIs. In order to control the input light polarization, a polarization controller (PC) is connected to the sensing fiber. The signal at the output port of the fiber coupler is a result of interference between the beams polarized along the slow axis and the fast axis. The phase of the interference is simply given as

$$
\delta_{S L}=\frac{2 \pi}{\lambda} B L
$$

where

$B=\left|n_{f}-n_{s}\right|$ is the birefrigent coefficient of the sensing fiber

$L$ is the length of the fiber

$n_{f}$ and $n_{s}$ are the effective indices of the fast and slow modes

Fiber-optic-based SIs can be used for measuring parameters such as strain, temperature, pressure, and twist. Such fiber-optic-based SIs provide the value of the sensed parameter averaged over the length of the sensor. However, a disadvantage of the SI is its significant temperature and strain cross-sensitivity. Rajan et al. reported that the temperature strain cross-sensitivity issue can be eliminated by employing PM-PCFs [58]. The output of a low temperature-sensitive PM-PCF SI-based sensor embedded in composite beam undergoing a three-point bending deflection is shown in Figure 17.17. Such low temperature-sensitive HB fiber-based SIs are an appropriate option for measuring strain acting on a composite material over the length of the sensing fiber.

\subsubsection{Optical Fiber Polarimetric Sensors for Composite Materials}

The polarization properties of light propagating through an optical fiber can be affected by stress, strain, pressure, and temperature acting on a measuring fiber, and in a fiber polarimetric sensor, the polarization change is detected to retrieve the sensing parameter $[59,60]$. A symmetric deformation effect or temperature variation in a single-mode fiber influences the propagation constant $(\beta)$ for every mode because of the changes in the fiber length $(L)$ and the refractive indices of the core and

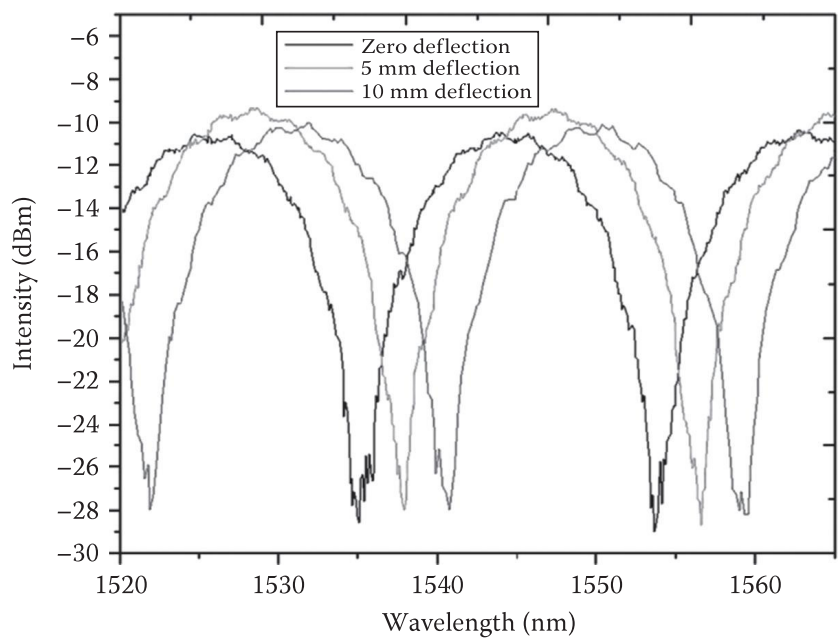

FIGURE 17.17 Responses of the PM-PCF SI-based sensor embedded in the composite material sample during deflections based on a three-point bending test. 
the cladding $[24,61]$. Under the influence of longitudinal strain $(\varepsilon)$ or temperature $(T)$, for singlemode fiber polarimetric sensors, the change in the phase difference can be written as [61]

$$
\frac{\delta(\Delta \Phi)}{\delta X}=\Delta \beta \frac{\partial L}{\partial X}+L \frac{\partial(\Delta \beta)}{\partial X}
$$

where $X$ stands for temperature, pressure, or strain.

Optical fiber polarimetric sensors can be realized by different types of PM fibers such as Panda fiber, bow-tie-type fiber, side-hole fibers, and PM-HB MOF fibers. Optical fiber polarimetric sensors can be embedded in composite materials to measure the average strain/temperature over the sensor length [24,61]. It is possible to vary the strain/temperature sensitivity of optical fiber polarimetric sensors by selecting a PM fiber type with appropriate birefringence and length [61,62].

For optical fiber polarimetric sensors, the phase difference between the two orthogonal polarizations can be extracted using an experimental setup consisting of a tunable laser source and a polarimeter/polarization control system [63]. Optical fiber polarimetric sensors can also be operated in intensity domain with the help of a polarizer-analyzer arrangement, and the experimental setup for operation in intensity domain is shown in Figure 17.18. For polarimetric sensors, the change in the output intensity at a wavelength $\lambda$ due to externally applied longitudinal strain can be described by the following formula [24,61]:

$$
I_{s}(\lambda)=\frac{I_{0}}{2}[1+\cos (\Delta \Phi)]
$$

For such polarimetric sensors operating in the intensity domain, periodic variations in the output intensity can be associated with applied strain or temperature [64]. Woliński et al. reported that low strain-sensitive polarimetric sensors such as PM-PCF polarimetric sensor guarantee a linear response for a wide range of applied strain values [61] (Figure 17.19). Moreover, the insignificant temperature sensitivity of PM-PCFs makes them the most appropriate candidates for composite strain measurements [64]. However, for local strain/temperature measurements of large composite structures, polarimetric sensors cannot be employed as the polarimetric sensors measures average strain over the length of the sensing fiber.

When embedded in the composite material, OFSs are influenced by various physical parameters of the composite material such as thermal expansion composite materials [14]. It is found that PM-PCF polarimetric fiber sensor with an acrylate coating embedded in the composite material shows the same response as the one in free space while the coating-stripped fiber polarimetric sensor shows significant temperature sensitivity when embedded in the composite material as shown in Figure 17.20a and b. This is due to the stress-induced change in birefringence created by the thermal expansion of the composite material, while in the case of a buffer-coated fiber, the effect is considerably reduced as the thermal stress is largely eliminated by the buffer coating. While a coating-stripped fiber polarimetric sensor shows a significant increase in temperature sensitivity when embedded in the composite material as shown in Figure 17.20c and d, which is due to the stress-induced change in birefringence created by thermally induced strain. A comparison of

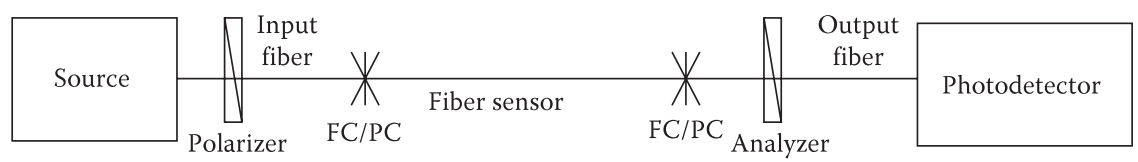

FIGURE 17.18 Experimental setup for measurements with fiber-optic polarimetric sensors in intensity domain. 


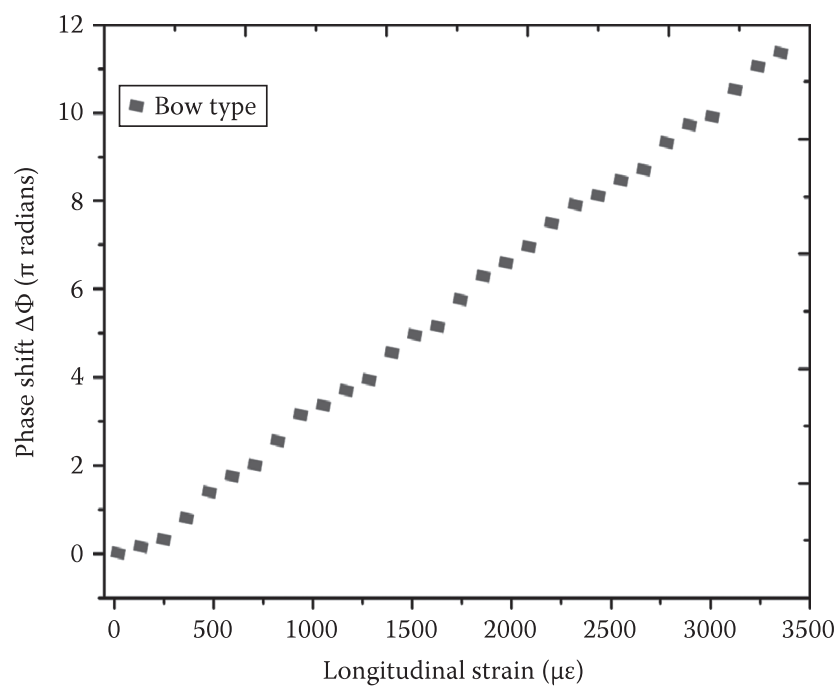

FIGURE 17.19 Change of the polarization of fiber-optic polarimetric sensors as a function of strain applied to a composite sample. (From Domański, A.W. et al., Photon. Lett. Poland, 1, 121, 2009.)

temperature sensitivities of PM-PCF sensors in various configurations (with and without buffer coating) at different layers is shown in Figure 17.20e, and it is clear that the presence of a coating preserves the temperature independence of a PM-PCF sensor when it is embedded in a composite. The thermal expansion of the composite material is the main source of error in strain and temperature measurements using embedded polarimetric fiber sensors, and more accurate strain and temperature measurements can be obtained with buffer-coated polarimetric fiber sensors.

It is also found that the temperature-induced phase change of buffer stripped PM-PCF polarimetric sensors embedded in different type composite material samples varies with the amount of thermal elongation induced strain over a temperature for the particular material. Figure 17.21 shows the observed phase shift for stripped PM-PCF sensors embedded in different composite samples for temperature variation from $0^{\circ} \mathrm{C}$ to $65^{\circ} \mathrm{C}$. The information from embedded sensors opens up the possibility of measuring the CTE value of a composite material itself, based on the observed phase change of the embedded buffer stripped PM-PCF polarimetric sensor. The CTE of carbon-epoxy, E glass-epoxy, and E glass-unsaturated polyester resin composite material samples is measured experimentally using the PM-PCF sensor and it shows good agreement with the theoretically calculated CTE values as shown in Figure 17.22. Thus, PM-PCF-based polarimetric sensor can provide a vital key information for the composite manufacturing industry by further developing nondestructive methods based on embedded OFSs for the characterization of composite material at different stages in their life cycle, from fabrication through operation to predicting the onset of failure.

\subsubsection{Distributed Optical Fiber Sensors}

Distributed optical fiber sensors (DOFSs) are capable of providing a continuous measurand profile over the length of the optical fiber and thus are very promising for strain/temperature measurements in large structures such as bridges, buildings, and pipelines $[25,65,66]$. However, given the nature of composite structures, the length is normally limited to $80 \mathrm{~m}$ or less with a strain or temperature requirement of at least $1^{\circ} \mathrm{C}$ or $20 \mu \varepsilon$ with a few centimeters resolution. DOFSs are categorized into several types based on the sensing technology and the physical effect underpinning the operating principle: (1) optical time-domain reflectometry (OTDR) and optical frequency-domain reflectometry (OFDR), both based on Rayleigh scattering; (2) Raman optical time-domain reflectometry 


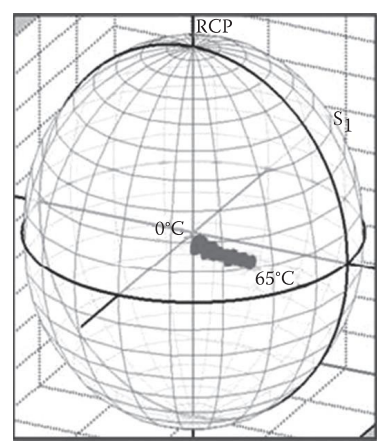

(a)

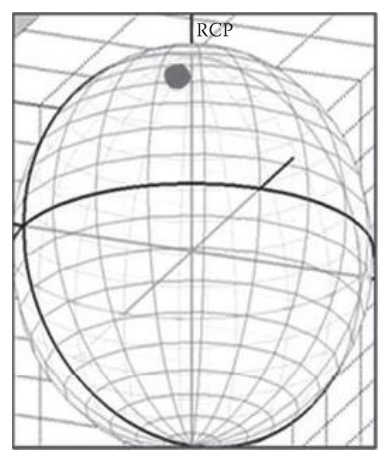

(c)

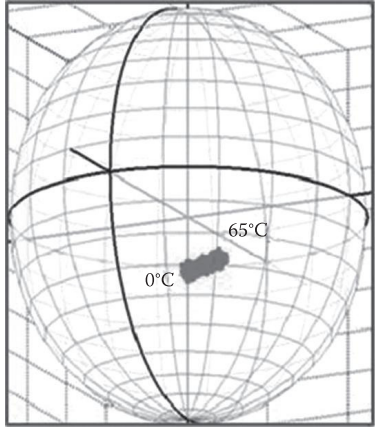

(b)

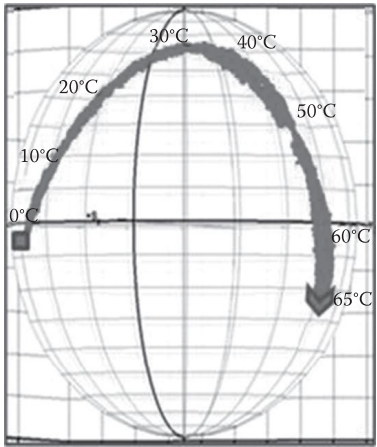

(d)

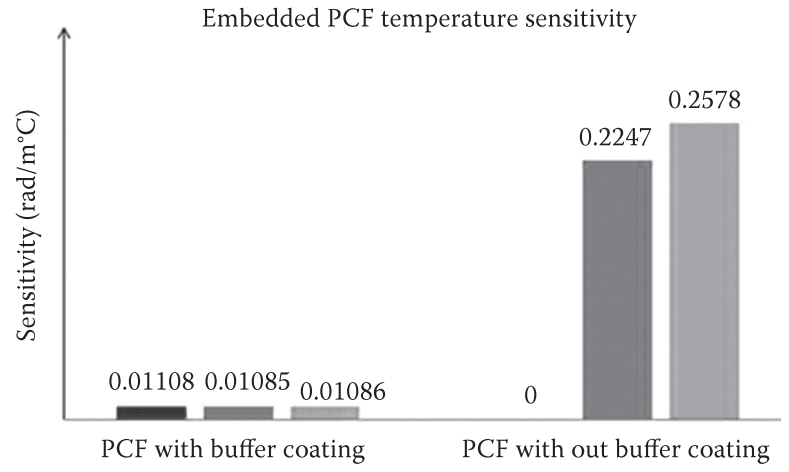

Gree space $\quad$ Layer 5 Layer 1

FIGURE 17.20 (a) Observed phase shift in coated PM-PCF fibers for a temperature variation from $0^{\circ} \mathrm{C}$ to $65^{\circ} \mathrm{C}$ (a) free space (b) after embedding. Observed phase shift in coating-stripped PM-PCF fibers for a temperature variation from $0^{\circ} \mathrm{C}$ to $65^{\circ} \mathrm{C}$ (c) in free space (d) after embedding and (e) comparison of the temperature sensitivity of $\mathrm{PM}-\mathrm{PCF}$ polarimetric sensors at different configurations.

(ROTDR) and Raman optical frequency-domain reflectometry (ROFDR), both based on Raman scattering; and (3) Brillouin optical time-domain reflectometry (BOTDR) and Brillouin optical frequency-domain reflectometry (BOFDR), both based on Brillouin scattering [67].

OTDR and OFDR are the first generation of optical fiber distributed sensors, based on the use of Rayleigh scattering to reflect the attenuation profiles of long-range optical fiber links [68]. An example of strain measurement by OFDR technique for various loading conditions is shown in Figure 17.23. An optical pulse is launched into an optical fiber and the power of the Rayleigh 


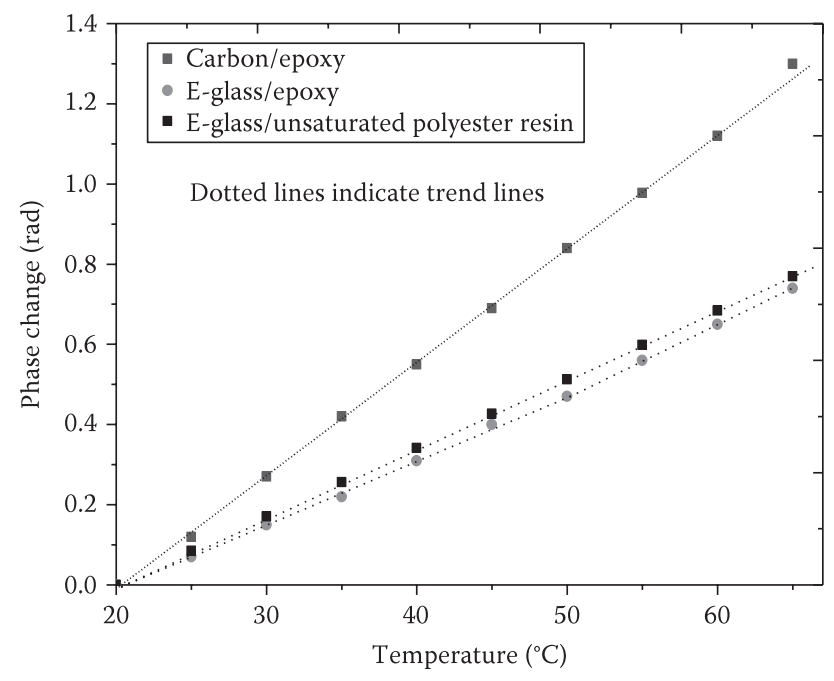

FIGURE 17.21 Observed phase shift for stripped PM-PCF sensors embedded in different composite samples for temperature variation from $0^{\circ} \mathrm{C}$ to $65^{\circ} \mathrm{C}$.

backscattered light is detected using a photodetector as the light pulse propagates along the fiber link [67]. Frequency-based Brillouin method can provide rapid strain/temperature sensing [69]. BOFDR can be employed to measure strain/temperature variations as small as $1^{\circ} \mathrm{C}$ or $20 \mu \varepsilon$ with a few centimeters of resolution [69,70]. For BOFDR-based long-distance measurements, singlemode fibers are used [69,71]. ROTDR is an appropriate candidate for temperature measurements in composites since the intensity ratio between anti-Stokes components and Stokes components of the ROTDR response provides information about temperature [71]. ROTDR can be employed to measure temperature variations as small as $0.1^{\circ} \mathrm{C}$ with a few meters of resolution [71,72]. Multimode fibers are commonly used for ROTDR-based short-distance measurements [72]. In the case of composite strain/temperature measurements from the aforementioned categories of DOFS, an appropriate sensing technology can be selected based on the application and its requirements; specifically, BOFDR is more suitable for strain measurements and ROTDR is more suitable for temperature measurements in composite material [71-73].

\subsubsection{Hybrid Sensing Approaches for Simultaneous Strain and Temperature Measurements in Composite Materials}

In most of practical applications of composite materials, the key requirement is to measure more than one parameter. For example, for SHM applications in composite parts such as helicopter rotor blades, wind turbines, and aircraft structures, a simultaneous monitoring of temperature and strain is favorable. Several OFSs such as FBGs and PM polarimetric sensors are capable of measuring strain and temperature, but there is always an issue of accurate discrimination between these two influencing factors $[23,25]$. One of the simplest and promising methods for simultaneous measurement of strain and temperature in composite parts is the hybrid optical fiber sensing approach; in this, two or more OFSs operate in a combined manner to eliminate the disadvantages of individual OFSs providing accurate and independent strain/temperature information [74]. Many researchers have investigated different hybrid sensing approaches for simultaneous strain and temperature measurements such as using a combination of FBG sensors with various types of sensors such as LPGs [36,74], Fabry-Perot interferometer sensors [37], PCF modal interferometers [75], and FLMs using a small-core MOF [76]. Sensors based on gratings written in MOFs and standard 

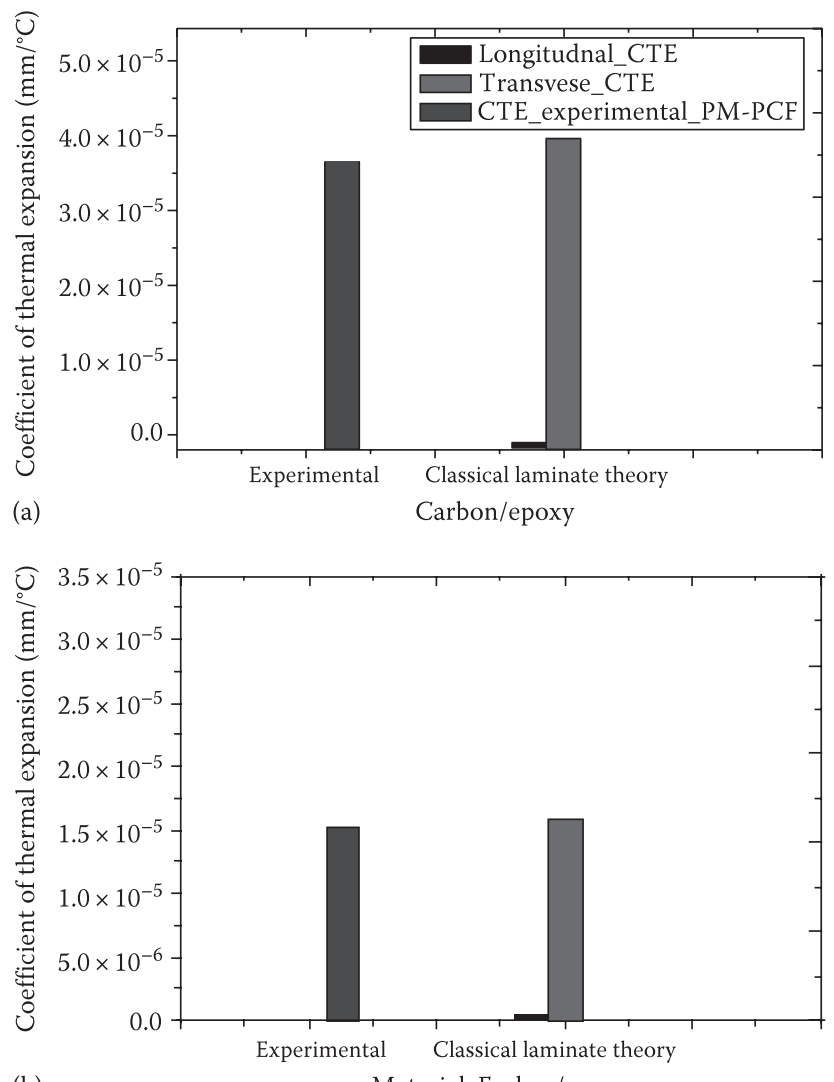

(b) Material: E-glass/epoxy

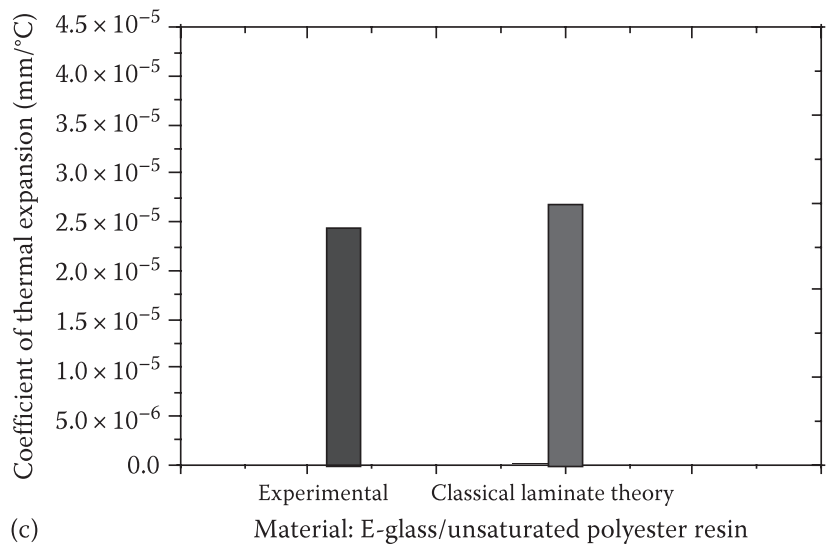

FIGURE 17.22 Comparison of theoretical predictions with experiment, (a) carbon-epoxy and (b, c) E glassepoxy/E glass-resin composite samples.

optical fibers have also been reported for discrimination between strain and temperature [77]. Previously, we reported a hybrid approach that involves an FBG sensor and a PM-PCF polarimetric sensor for simultaneous measurement of strain and temperature [78] of composite materials. In the context of composite structures, the temperature sensitivity of an OFS is largely influenced by the thermal strain of the composite material [14]. Sensors based on stripped PM-PCF-based 


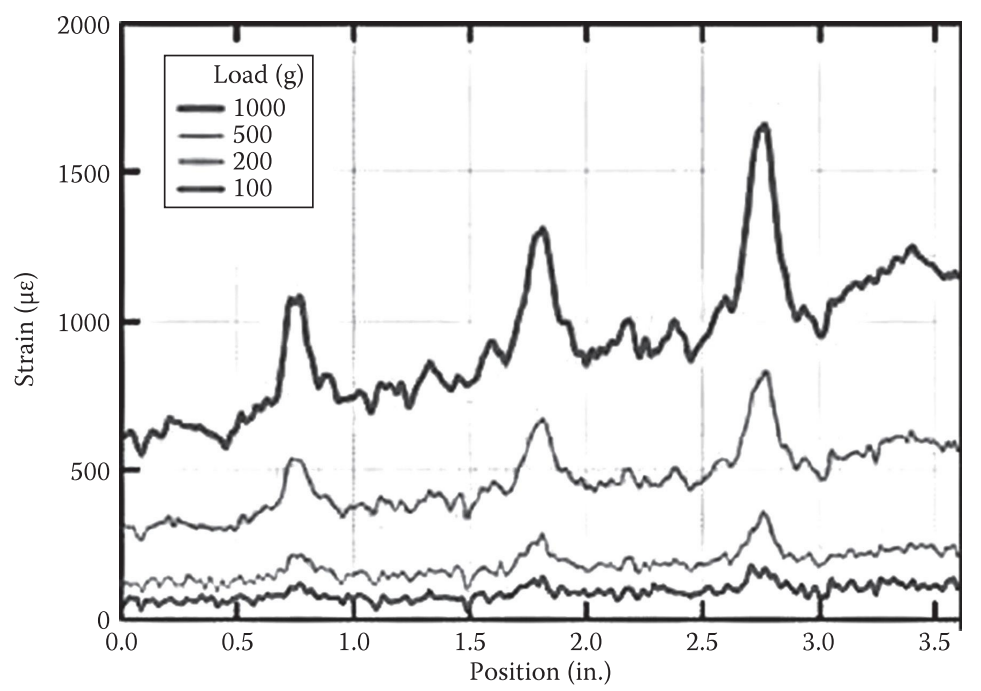

FIGURE 17.23 Strain measured by the optical fiber bonded to a composite sample for various loading conditions. (Courtesy of Luna Engineering Note EN-FY1317.)

polarimetric sensors have been reported for thermal strain and thermal expansion measurements in composite materials [13]. A hybrid OFS embedded in a composite material that is capable of discriminating between strain, temperature, and thermal strain has great potential for future OFSbased SHM applications.

\subsection{LIGHTWEIGHT SANDWICH COMPOSITES WITH EMBEDDED FIBER SENSORS}

Among the different types of composite material laminates, it is common in advanced manufacturing industries to construct composite laminates using a sandwich construction, consisting of an outer layer of reinforcing fiber in conjunction with a core filling, such as a solid foam core or a honeycomb core [79]. The combination of carbon fiber-reinforced composite materials with structurally efficient sandwich laminate design offers novel opportunities for ultra-lightweight structures. This sandwich combination allows the structural element with a very high bending stiffness to weight ratio as well as bending strength to weight ratio [80].

Many applications including marine and automobile industry [81] use sandwich composite construction to save weight and increase stiffness, because the low density foam is lightweight while ensuring that the reinforcing fibers sustain the majority of the stress at the extremes from the neutral bending axis. However, one of the main concerns related to sandwich composite structures is the fact that their load carrying ability can be significantly reduced by the presence of delamination between the foam core and the reinforcement fiber. Such damages usually cannot be observed from outside the sandwich construction but can cause a serious reduction in load-bearing capacity of the structure. For sandwich structures, due to the presence of the core material, the performance of the embedded sensor could be different from those of the standard composite laminates.

FBG embedded in sandwich composites are used to measure strain, impact, and temperature of the composites. Rajan et al. demonstrated a carbon foam core sandwich composite (Figure 17.24) embedded with composite materials and the strain experienced within the material is measured [82]. Other works carried out by Peters et al. showed that FBGs can be used to impact measurement in foam core sandwich composites [83]. Rajan et al. also demonstrated the temperature measurements of sandwich composites using embedded FBGs. The measured wavelength shift for the embedded 


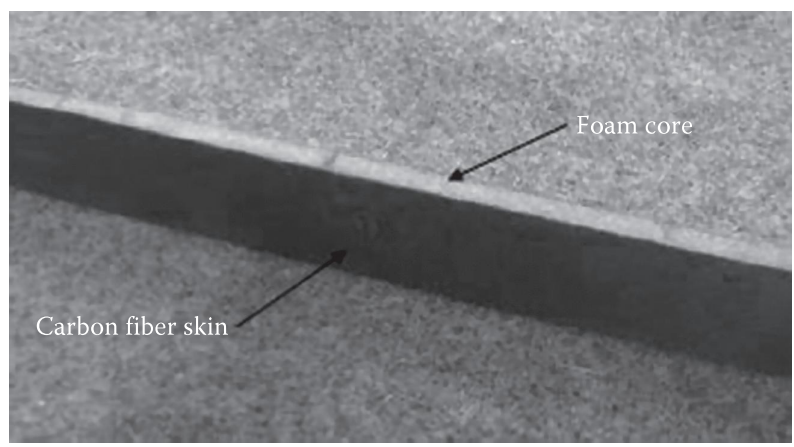

FIGURE 17.24 A carbon fiber foam core sandwich composite.

FBG with change in temperature is shown in Figure 17.25a. From the graph, it can be seen that the carbon fiber foam composite specimen shows a temperature sensitivity of approximately $9.5 \mathrm{pm} /{ }^{\circ} \mathrm{C}$, which is close to the free-space sensitivity of the $\mathrm{FBG}\left(10 \mathrm{pm} /{ }^{\circ} \mathrm{C}\right)$. In comparison with embedded FBGs in standard glass and carbon fiber composites, which shows considerably high sensitivity $\left(12-20 \mathrm{pm} /{ }^{\circ} \mathrm{C}\right)[14]$ due to the stress/strain induced by the thermal expansion of the composite, the results obtained for the carbon foam composite is highly promising, as there was no considerable effect of the material properties on the sensitivity of the embedded grating, and hence the embedded sensor can accurately measure the true temperature inside the composite.

Another important factor to be considered in the case of a foam core sandwich composite is the relaxation of the foam during bending measurements and its effects on the performance of the embedded FBG. To evaluate this, the reflection spectra of the FBG are monitored after removing the physical pressure applied to the laminate. It was observed that the FBG peak reflected wavelength continues to shift slightly even after the complete removal of the load as shown in Figure $17.25 \mathrm{~b}$. This is assumed to be due to the relaxation of the foam core in the composite sandwich. The observed wavelength shift was approximately $0.02 \mathrm{~nm}$ for a time period of $60 \mathrm{~s}$, which is equivalent to a strain of $16 \mu \varepsilon$ given a strain sensitivity of $12 \mathrm{pm} / \mu \varepsilon$. This adds an error of $\pm 2.5 \%$ in the strain measurement using the embedded FBG in the case of the sandwich laminates. However, for the foam relaxation, the percentage of error also depends on the amount of load applied and the type of load applied. Further research is required to quantitatively predict the suitability of using embedded FBG for accurate impact/strain and temperature measurement for sandwich composites, and many groups are currently focusing on SHM of these types of lightweight composites.
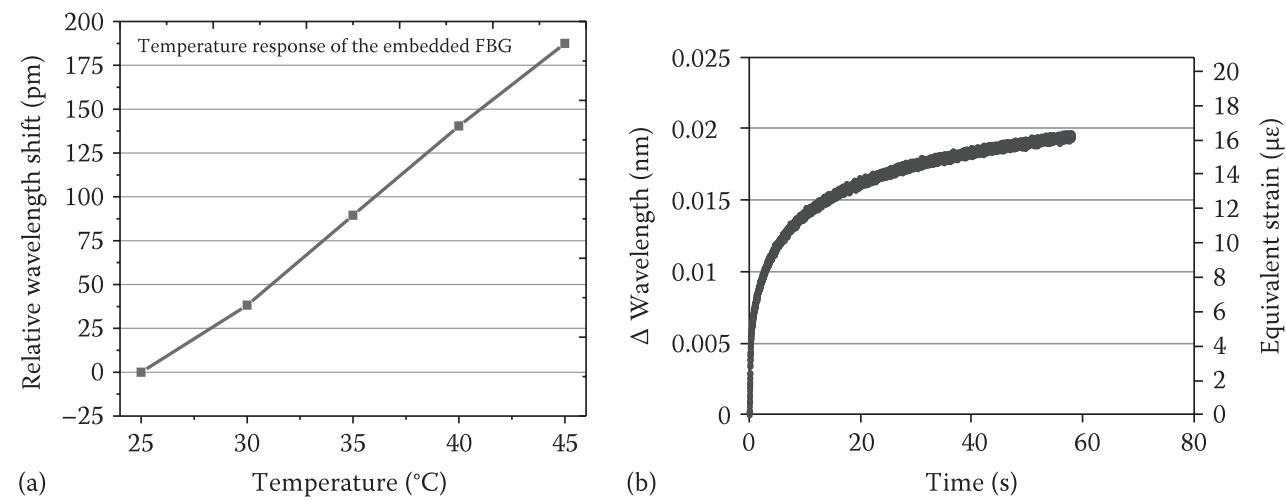

FIGURE 17.25 (a) Temperature response of the embedded FBG in a sandwich composite and (b) wavelength shift of the FBG due to the gradual relaxation of the foam core. 


\subsection{RECENT TRENDS, ISSUES, AND FUTURE CHALLENGES OF THE OFS TECHNOLOGY}

Embedding an optical fiber inside composite materials is a minimally invasive technique; however, for industrial applications, there still exist a few issues that are under investigation and in this section we discuss those challenging issues. One of the challenges with composite structures embedded with a fiber sensor is the provision for a reliable method for accessing the sensor $[84,85]$. Custom-designed connectors with ingress/egress optical fiber ends are a promising technique, and one example of such a connector is shown in Figure 17.26. However, such egress optical fibers may become brittle at the edges of the smart composite structures [21,86]. Also, connecting to such a connector is very hard and edge trimming of the composite after the connector installation is impossible.

The second issue is the structural damage to the composite material due to the fact that optical fibers have larger diameter compared to reinforcement fibers. As a solution, researchers are considering new optical fibers with a reduced diameter and an optimized coating. One example of such fibers is a thinner optical fiber with $80 \mu \mathrm{m}$ diameter known as Draw Tower Grating (DTG) fiber, which is proven to be less invasive when embedded within laminate composite structures or textiles [87].

Another issue related to embedding of fiber sensor in a composite part in weight- or spacesensitive applications is the large size of conventional OFS interrogators, which could present difficulties for sensing of composite parts that, for example, are in constant motion, such as helicopter rotor blades and wind turbine structures [88]. To an extent, this issue can be resolved by adopting miniaturized interrogation modules based on photonic integrated circuits (PIC) or flexible polymer waveguides $[89,90]$. Such surface attachable flexible interrogators allow for integration of photodetector arrays with wireless communication technology and thus have strong potential in smart sensing of composite parts in motion.

Finally, it must be recognized that the OFS embedding procedure is often a labor-intensive task. Therefore, ideally it requires a reliable automated optical fiber placement system that matches well with the existing industrial composite production processes. Several composite manufactures, such as Airborne, have introduced automated fiber placement system that provide control over embedding depth, prestrain, position, and alignment. Such an all-automated fiber placement system is shown in Figure 17.27. The biggest challenge in using most of the automated fiber sensor embedding systems is that it is difficult to ensure an adequate repeatability of the fiber sensor placement. In addition, during such an automated fabrication process, the safety of delicate areas of optical fiber (such as grating written area of FBG, spliced and buffer coating removed areas of sensing optical fiber) and control over the alignment of specialized fibers such as MOFs are not well assured. Thus,

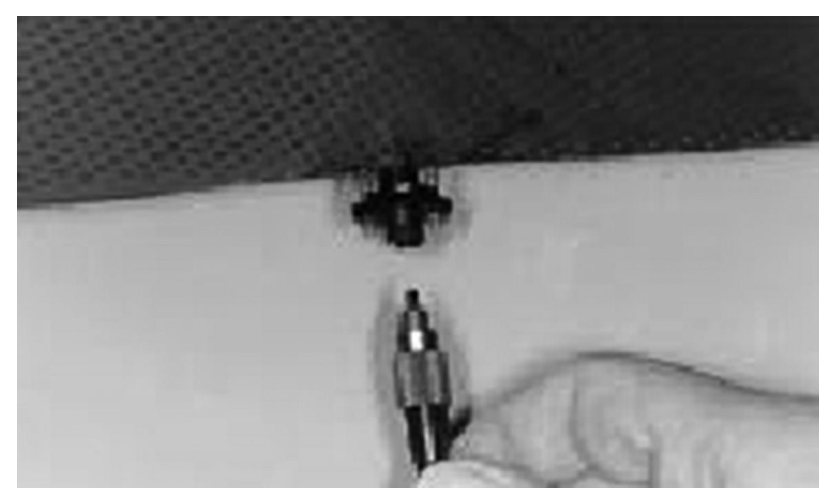

FIGURE 17.26 Egress optical fiber with connector. 


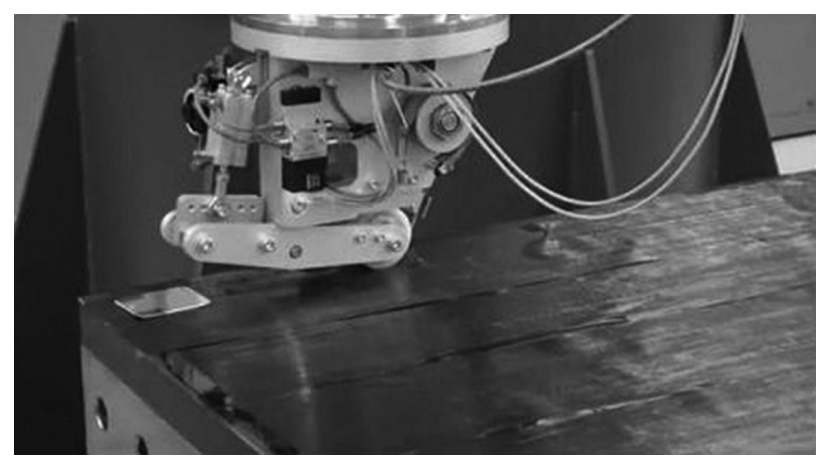

FIGURE 17.27 Automated fiber placement system. (Courtesy of Airborne: the future in composites.)

an automated fiber placement system together with an X-ray-based microcontrolled tomography could be a suitable solution for quality control of the embedded fiber sensor and a realistic option for appropriate alignment of optical fibers for future smart sensing applications.

\subsection{CONCLUSION}

This chapter presents a comprehensive overview of the OFSs used for sensing of composite materials and provides essential information regarding many aspects, including different types of composite materials, properties, and their performance, the relevance of OFS for composite material sensing applications, future challenges of embedded OFSs, and recent trends. Embedded OFSs are useful to the smart sensing applications of composite materials in aerospace, structural, civil, and sports industry and this technology will be commercially available and beneficial to the society after a few more years of research work.

\section{REFERENCES}

1. Garg, D. P., Zikry, M. A., Anderson, G. L., Gros, X. E., Current and potential future research activities in adaptive structures: An ARO perspective, Smart Materials and Structures, 10, 610-623, 2001.

2. Balageas, D., Introduction to structural health monitoring, ISTE, pp. 16-43, 2006.

3. Jian, C., Lei, Q., Shenfang, Y., Lihua, S., PeiPei, L., Dong, L., Structural health monitoring for composite materials, in $\mathrm{Hu}, \mathrm{N}$. (ed.), Composites and Their Applications, InTech, Rijeka, Croatia, 2012. ISBN: 978-953-51-0706-4.

4. Zhu, Y. K., Gui, Y. T., Rong, S. L., Hong, Z., A review of optical NDT technologies, Sensors, 11, 77737798, 2011.

5. Gros, X. E., Current and future trends in non-destructive testing of composite materials, Annales de Chimie Science Matériaux, 25, 539-544, 2000.

6. Błażejewski, W., Gąsior, P., Kaleta, J., Application of optical fibre sensors to measuring the mechanical AQ12 properties of composite materials and structures, in Advances in Composite Materials: Ecodesign and Analysis, InTech, Rijeka, Croatia, 2011. ISBN: 978-953-307-150-3.

7. Méndez, A., Csipkes, A., Overview of fibre optic sensors for NDT applications, in Nondestructive Testing of Materials and Structures, Springer, Dordrecht, the Netherlands, 2013, pp. 179-184.

8. Chung, D. D. L., Composite Materials: Science and Applications, 2nd edn., Springer Publications, London, U.K., 2010.

9. Kaw, A. K., Mechanics of Composite Materials, CRC Press, Boca Raton, FL, 2010.

10. Bunsell, A. R., Jacques, R., Fundamentals of Fibre Reinforced Composite Materials, CRC Press, Boca Raton, FL, 2010.

11. Eaton, M. J., Pullin, R., Holford, K. M., Acoustic emission source location in composite materials using delta T mapping, Composites Part A, 43(6), 856-863, 2012.

12. Elmar, W., Bernhard, J., Composites market report, The European GRP market (AVK), The global CRP AQ13 market (CCeV), 2013. 
13. Ramakrishnan, M., Rajan, G., Semenova, Y., Boczkowska, A., Domański, A., Wolinski, T., Farrell, G., Measurement of thermal elongation induced strain of a composite material using a polarization maintaining photonic crystal fibre sensor, Sensors and Actuators A: Physical, 190, 44-51, 2013.

14. Ramakrishnan, M., Rajan, G., Semenova, Y., Lesiak, P., Domanski, A., Wolinski, T., Boczkowska, A., Farrell, G., The influence of thermal expansion of a composite material on embedded polarimetric sensors, Smart Materials and Structures, 20, 125002, 2011.

15. Lesiak, P. et al., Influence of lamination process on optical fibre sensors embedded in composite material, Measurement, 45, 2275-2280, 2012.

16. Jensen, D. W., Pascual, J., Degradation of graphite/bismaleimide laminates with multiple embedded fibre-optic sensors, Proceedings of SPIE, 1370, 228-237, 1990.

17. Lee, D. C., Lee, J. J., Yun, S. J., The mechanical characteristics of smart composite structures with embedded optical fibre sensors, Composite Structure, 32, 39-50, 1995.

18. Güemes, J., Perez, J. S., Fibre Optics Sensors in New Trends in Structural Health Monitoring, Springer, Vienna, Austria, 2013, pp. 265-316.

19. Emmanwori, L., Shivakumar, K. N., Structural performance of composite laminates with embedded fibre optic sensor under tension and compression loads, Proceedings of the 43rd Annual Conference of American Institute of Aeronautics and Astronautics, Denver, CO, 2002.

20. Ciang, C. C., Jung, R. L., Hyung, J. B., Structural health monitoring for a wind turbine system: A review of damage detection methods, Measurement Science and Technology, 19, 122001, 2008.

21. Zhou, G., Sim, L. M., Damage detection and assessment in fibre-reinforced composite structures with embedded fibre optic sensors: Review, Smart Materials and Structures, 11, 925-939, 2002.

22. Du, W., Tao, X. M., Tam, H. Y., Choy, C. L., Fundamentals and applications of optical fibre Bragg grating sensors to textile structural composites, Composite Structures, 42, 217-229, 1998.

23. Rao, Y. J., Recent progress in fibre-optic extrinsic Fabry-Perot interferometric sensors, Optical Fibre Technology, 12, 227-237, 2006.

24. Murukeshan, V. M., Chan, P. Y., Ong, L. S., Asundi, A., On-line health monitoring of smart composite structures using fibre polarimetric sensor, Smart Materials and Structures, 8, 544-548, 1999.

25. Murayama, H., Kazuro, K., Hirosh, N., Akiyoshi, S., Kiyoshi, U., Application of fibre-optic distributed sensors to health monitoring for full-scale composite structures, Journal of Intelligent Material Systems and Structures, 14, 3-13, 2003.

26. Lopez-Higuera, J. M., Cobo, L. R., Incera, A. Q., Cobo, A., Fibre optic sensors in structural health monitoring, Journal of Lightwave Technology, 29(4), 587-608, 2011.

27. Gerard, F. F., Fibre optic sensor systems for monitoring composite structures, RP Asia 2005 Conference, Bangkok, Thailand, August 25-26, 2005.

28. Montanini, R., D'Acquisto, L., Simultaneous measurement of temperature and strain in glass fibre/epoxy composites by embedded fibre optic sensors: I. Cure monitoring, Smart Materials and Structures, 16, $1718,2007$.

29. Glisic, B., Inaudi, D., Fibre Optic Methods for Structural Health Monitoring, 1st edn., John Wiley \& Sons, Ltd., Chichester, U.K., 2007.

30. Hill, K. O., Meltz, G., Fibre Bragg grating technology fundamentals and overview, Journal of Lightwave Technology, 15, 1263-1276, 1997.

31. Raman, K., Fibre Bragg Gratings, Academic Press, San Diego, CA, 1999.

32. Yashiro, S., Okabe, T., Toyama, N., Takeda, N., Monitoring damage in holed CFRP laminates using embedded chirped FBG sensors, International Journal of Solids and Structures, 44(2), 603-613, 2007.

33. Dong, X., Zhang, H., Liu, B., Miao, Y., Tilted fibre Bragg gratings: Principle and sensing applications, Photonic Sensors, 1(1), 6-30, 2011.

34. Zhao, Y., Yanbiao, L., Discrimination methods and demodulation techniques for fibre Bragg grating sensors, Optics and Lasers in Engineering, 41, 1-18, 2004.

35. Xu, M. G., Archambault, J. L., Reekie, L., Dakin, J. P., Discrimination between strain and temperature effects using dual-wavelength fibre grating sensors Electronics Letters, 30, 1085-1087, 1994.

36. James, S. W., Dockney, M. L., Tatam, R. P., Simultaneous independent temperature and strain measurement using in-fibre Bragg grating sensors, Electronics Letters, 32, 1133-1134, 1996.

37. Samer, K. B., Sun, T., Grattan, K. T. V., Simultaneous measurement of temperature and strain with long period grating pairs using low resolution detection, Sensors and Actuators, 144, 83-89, 2008.

38. Oliveira, R. D., Ramos, C. A., Marques, A. T., Health monitoring of composite structures by embedded FBG and interferometric Fabry-Perot sensors, Computers and Structures, 86, 340-346, 2008.

39. Guan, B. O., Tam, H. Y., Tao, X. M., and Dong, X. Y., Simultaneous strain and temperature measurement using a superstructure fibre Bragg grating, IEEE Photonics Technology Letters, 12, 675-677, 2000. 
40. Luyckx, G., Eli, V., Nicolas, L., Joris, D., Strain measurements of composite laminates with embedded fibre Bragg gratings: Criticism and opportunities for research, Sensors, 11, 384-408, 2011.

41. Sonnenfeld, C., Sulejmani, S., Geernaert, T., Eve, S., Lammens, N., Luyckx, G., Eli, V., Thienpont, H., Microstructured optical fibre sensors embedded in a laminate composite for smart material applications, Sensors, 11, 2566-2579, 2011.

42. Reid, S. R., Impact Behaviour of Fibre-Reinforced Composite Materials and Structures, CRC Press, Boca Raton, FL, 2003.

43. Rajan, G., Ramakrishnan, M., Semenova, Y., Boczkowska, A., Domanski, A., Woliński, T., Farrell, G., Analysis of vibration measurement in composite materials using embedded PM-PCF polarimetric sensor and an FBG sensor, IEEE Sensors Journal, 12(5), 1365-1371, 2012.

44. Rajan, G., Ramakrishnan, M., Semenova, Y., Farrell, G., Performance analysis of composite materials AQ14 embedded with optical fibre sensors, Proceedings of the IEEE Sensors, 351-354, 2011.

45. Azmi, A. I., Peng, G.-D., Raju, Failure monitoring of E-glass/vinylester composites using fibre grating AQ15 acoustic sensor, Photonic Sensors, 3(2), 184-192, 2013.

46. Luyckx, G. et al., Response of FBGs in microstructured and bow tie fibres embedded in laminated composite, IEEE Photonics Technology Letters, 21, 1290-1292, 2009.

47. Peng, G.-D., Xiong, Z., Chu, P. L., Photosensitivity and gratings in dye-doped polymer optical fibres, Optical Fibre Technology, 5, 242-251, 1999.

48. Xiong, Z., Peng, G.-D., Wu, B., Chu, P. L., Highly tunable Bragg gratings in single-mode polymer optical fibres, IEEE Photonics Technology Letters, 11, 352-354, 1999.

49. Kuang, K. S. C., Quek, S. T., Koh, C. G., Cantwell, W. J., Scully, P. J., Plastic optical fibre sensors for structural health monitoring: A review of recent progress, Journal of Sensors, 2009, 312053, 2009.

50. Webb, D. J., Polymer optical fibre Bragg gratings, in Bragg gratings, photosensitivity and poling in glass waveguides, OSA Technical Digest, Paper Btu3E.4.4, 2012.

51. Kalli, K., Dobb, H. L., Webb, D. J., Carroll, K., Themistos, C., Komodromos, M., Peng, G.-D., Fang, Q., Boyd, I. W., Development of an electrically tuneable Bragg grating filter in polymer optical fibre operating at $1.55 \mu \mathrm{m}$, Measurement Science and Technology, 17, 3155-3164, 2007.

52. Zhang, W., Webb, D. J., Peng, G.-D., Investigation in to time response of polymer fibre Bragg grating based humidity sensors, Journal of Lightwave Technology, 30, 1090-1096, 2012.

53. Rajan, G., Ramakrishnan, M., Semeonva, Y., Ambikairajah, E., Farrell, G., Peng, G.-D., Experimental study and analysis of a polymer fibre Bragg grating embedded in a composite material, Journal of Lightwave Technology, 32(9), 1726-1733, 2014.

54. Lee, B. H., Kim, Y. H., Park, K. S., Eom, J. B., Kim, M. J., Rho, B. S., Choi, H. Y., Interferometric fibre optic sensors, Sensors, 12, 2467-2486, 2012.

55. Liu, T., Wu, M., Rao, Y., Jackson, D. A., Fernando G. F., A multiplexed optical fibre-based extrinsic Fabry-Perot sensor system for in-situ strain monitoring in composites, Smart Materials and Structures, 7, 550, 1998

56. Leng, J. S., Asundi, A., Structural health monitoring of smart composite materials by using EFPI and FBG sensors, Sensors and Actuators A: Physical, 103, 330-340, 2003.

57. Fernando, G. F., Liu, T., Crosby, P., Doyle, C. A., Martin, D. B., Ralph, B., Badcock, R., A multi-purpose optical fibre sensor design for fibre reinforced composite materials Measurement Science and Technology, 8, 1065, 1997.

58. Rajan, G., Ramakrishnan, M., Lesiak, P., Semenova, Y., Wolinski, T., Boczkowska, A., Farrell, G., Composite materials with embedded photonic crystal fibre interferometric sensors, Sensors and Actuators A: Physical, 182, 57-67, 2012.

59. Wolinski, T. R., Polarimetric optical fibres and sensors, Progress in Optics, 40, 1-75, 2000.

60. Domanski, A. W., Tomasz, R. W., Wojtek, J. B., Polarimetric fibre optic sensors: State of the art and future, AQ16 International Conference on Interferometry-94, International Society for Optics and Photonics, 1994.

61. Wolinski, T. R., Lesiak, P., Domanski, A. W., Polarimetric optical fibre sensors of a new generation for industrial applications, Bulletin of the Polish Academy of Sciences, Technical Sciences, 56, 125-132, 2008.

62. Ma, Ji., Asundi, A., Structural health monitoring using a fibre optic polarimetric sensor and a fibre optic curvature sensor-static and dynamic test, Smart Materials and Structures, 10, 181-188, 2001.

63. Hogg, W. D., Roderick, D. T., Polarimetric fibre optic structural strain sensor characterization, AQ17 Proceedings of SPIE, 1170, 542-550, 1990.

64. Domański, A. W., Lesiak, P., Karolina, M., Boczkowska, A., Budaszewski, D., Ertman, S., Woliński, T. R., Temperature-insensitive fibre optic deformation sensor embedded in composite material, Photonics Letters of Poland, 1, 121-123, 2009.

65. Adachi, S., Distributed optical fibre sensors and their applications, IEEE SICE Annual Conference, 2008. 
66. Niklès, M., Fibre optic distributed scattering sensing system: Perspectives and challenges for high performance applications, Third European Workshop on Optical Fibre Sensor, International Society for Optics and Photonics, Napoli, Italy, 2007.

67. Grattan, K. T. V., Sun, T., Fibre optic sensor technology: An overview, Sensors and Actuators A: Physical, $82,40-61,2000$.

68. Kersey, A. D., A review of recent developments in fibre optic sensor technology, Optical Fibre Technology, 2, 291-317, 1996.

69. Bao, X., Liang, C., Recent progress in Brillouin scattering based fibre sensors, Sensors, 11, 4152-4187, 2011.

70. Hartog, A. H., Progress in distributed fibre optic temperature sensing, Proceedings of the Environmental and Industrial Sensing, International Society for Optics and Photonics, Chicago, IL, 2002.

71. Culshaw B., Optical fibre sensor technologies: Opportunities and-perhaps-pitfalls, Journal of Lightwave Technology, 22, 39-50, 2004.

72. Ravet, F., Distributed Brillouin sensor application to structural failure detection, in New Developments in Sensing Technology for Structural Health Monitoring, Springer, Berlin, Germany, 2011, pp. 93-136.

73. Zeng, X., Xiaoyi, B., Chia, Y. C., Theodore, W. B., Anthony, W. B., Michael, D. D., Graham, F., Alexander, L. K., Anastasis, V. G., Strain measurement in a concrete beam by use of the Brillouinscattering-based distributed fibre sensor with single-mode fibres embedded in glass fibre reinforced polymer rods and bonded to steel reinforcing bars, Applied Optics, 41, 5105-5114, 2002.

74. Patrick, H. J., Williams, G. M., Kersey, A. D., Pedrazzani, J. R., Hybrid fibre Bragg grating/long period grating sensor for strain/temperature discrimination, IEEE Photonics Technology Letter, 8, 1223-1225, 1996.

75. Dong, B., Hao, J., Liaw, C., Lin, B., Tjin, S. C., Simultaneous strain and temperature measurement using a compact photonic crystal fibre inter-modal interferometer and a fibre Bragg grating, Applied Optics, 49, 6232-6235, 2010.

76. Andre, R. M., Marques, M. B., Roy, P., Frazao, O., Fibre loop mirror using a small core micro-structured fibre for strain and temperature discrimination, IEEE Photonics Technology Letter, 22, 1120-1122, 2010.

77. Frazão, O., Carvalho, J. P., Ferreira, L. A., Araújo, F. M., Santos, J. L., Discrimination of strain and temperature using Bragg gratings in microstructured and standard optical fibres, Measurement Science and Technology, 16, 2109, 2005.

78. Rajan, G., Ramakrishnan, M., Semenova, Y., Karolina, M., Lesiak, P., Domanski, A. W., Wolinski, T. R., Farrell, G., A photonic crystal fibre and fibre Bragg grating-based hybrid fibre-optic sensor system, IEEE Sensors Journal, 12(5), 39-43, 2012.

79. Sokolinsky, V. S., Shen, H., Vaikhanski, L., Nutt, S. R., Experimental and analytical study of nonlinear bending response of sandwich beams, Composite Structures, 60, 219-2295, 2003.

80. Donaldson, S. L., Miracle, D. B., ASM Handbook Composites, Vol. 21, ASM International, Materials Park, OH, 2001.

81. Bakis, C. E. et al., Fibre-reinforced polymer composites for construction-state-of-the-art review, Journal of Composites for Construction, 6(2), 73-87, 2002.

82. Rajan, G., Darmanin, E., Peng, G.-D., Prusty, G., Carbon fibre-foam sandwich composite laminate embedded with fibre Bragg grating sensors, 23rd OFS Conference, Santander, Spain, 2014.

83. Hackney, D., Peters, K., Damage identification after impact in sandwich composites through embedded fibre Bragg sensors, Journal of Intelligent Material Systems and Structures, 22, 1305-1316, 2011.

84. Kang, H. K., Park, J. W., Ryu, C. Y., Hong, C. S., Kim, C. G., Development of fibre optic ingress/egress methods for smart composite structures, Smart Materials and Structures, 9, 149-156, 2000.

85. Green, A. K., Zaidman, M., Shafir, E., Tur, M., Gali, S., Infrastructure development for incorporating fibre-optic sensors in composite materials, Smart Materials and Structures, 9, 316-321, 2000.

86. Friebele E. J. et al., Optical fibre sensors for spacecraft applications, Smart Materials and Structures, 8, 813-838, 1999.

87. Chojetzki, C., Rothhardt, M., Ommer, J., Unger, S., Schuster, K., Mueller, H. R., High-reflectivity drawtower fibre Bragg gratings-Arrays and single gratings of type II, Optical Engineering, 44, 060503, 2005.

88. Palmer, D., Engelbart, R., Vaccaro, C., Future directions relative to NDE of composite structures, SAE Technical Paper 2004-01-2817, 2004.

89. Van, H., Bram, G. L., Erwin, B., Jeroen, M., Sandeep, K., Oliver, M., David, J. W., Kate, S., Peter, V. D., Geert, V. S., Ultra small integrated optical fibre sensing system, Sensors, 12, 12052, 2012.

AQ18 90. Missinne, J., Geert, V. S., Bram, V. H., Kristof, V. C., Tim, V. G., Peter, D., Jan, V., Peter, V. D., An array waveguide sensor for artificial optical skins, SPIE OPTO: Integrated Optoelectronic Devices, International Society for Optics and Photonics, 2009. 